\title{
Induced quantum metric fluctuations and the validity of semiclassical gravity
}

\author{
B. L. Hu and Albert Roura \\ Department of Physics, University of Maryland, College Park, Maryland 20742-4111, USA \\ Enric Verdaguer \\ Departament de Física Fonamental and CER en Astrofísica, Física de Partícules i Cosmologia, Universitat de Barcelona, \\ Av. Diagonal 647, 08028 Barcelona, Spain
}

(Received 6 February 2004; published 9 August 2004)

\begin{abstract}
We propose a criterion for the validity of semiclassical gravity (SCG) which is based on the stability of the solutions of SCG with respect to quantum metric fluctuations. We pay special attention to the two-point quantum correlation functions for the metric perturbations, which contain both intrinsic and induced fluctuations. These fluctuations can be described by the Einstein-Langevin equation obtained in the framework of stochastic gravity. Specifically, the Einstein-Langevin equation yields stochastic correlation functions for the metric perturbations which agree, to leading order in the large $N$ limit, with the quantum correlation functions of the theory of gravity interacting with $N$ matter fields. The homogeneous solutions of the Einstein-Langevin equation are equivalent to the solutions of the perturbed semiclassical equation, which describe the evolution of the expectation value of the quantum metric perturbations. The information on the intrinsic fluctuations, which are connected to the initial fluctuations of the metric perturbations, can also be retrieved entirely from the homogeneous solutions. However, the induced metric fluctuations proportional to the noise kernel can only be obtained from the Einstein-Langevin equation (the inhomogeneous term). These equations exhibit runaway solutions with exponential instabilities. A detailed discussion about different methods to deal with these instabilities is given. We illustrate our criterion by showing explicitly that flat space is stable and a description based on SCG is a valid approximation in that case.
\end{abstract}

DOI: 10.1103/PhysRevD.70.044002

PACS number(s): 04.62.+v, 03.65.Sq, 05.40.-a

\section{INTRODUCTION}

In this paper we discuss the conditions underlying the validity of semiclassical gravity (SCG), emphasizing the role of metric fluctuations induced by quantum matter sources. SCG is based on self-consistent solutions of the semiclassical Einstein equation for a classical spacetime driven by the expectation value of the stress tensor operator of quantum matter fields. We propose a criterion based on stochastic semiclassical gravity $[1,2]$ and compare it with a recently proposed criterion by Anderson et al. [3] based on linear response theory. To do this we need to reexamine all relevant factors old and new contributing to this issue, such as the reduction of higher derivative equations, intrinsic and induced fluctuations, and the relation between stochastic and quantum correlations. It also necessitates some clarification of the relation between our approach based on stochastic dynamics and the linear response approach and differences with the approach pursued by Ford and co-workers [4-11] based on the normal-ordering and integration-by-parts procedures on the stress-energy bitensor. The connection clarified and the bridges built in this process are beneficial to further development of "bottom-up" approaches to quantum gravity starting from SCG [12-15].

a. Metric fluctuations. SCG accounts for the averaged back reaction of quantum matter fields and can be regarded as a mean field approximation that describes the dynamics of the mean spacetime geometry. However, it does not account for the effects of the fluctuations of spacetime geometry, which can also be very important. Consider, for instance, the metric fluctuations induced by the vacuum fluctuations of the inflaton field in inflationary cosmological models. Those fluctuations play a crucial role in the generation of the primordial inhomogeneities which gave rise to the large scale structure of the present universe as well as the observed anisotropies of the cosmic microwave background of radiation.

This paper focuses on the effects of the quantum fluctuations of the metric. We will restrict our treatment to small metric perturbations around a given background geometry. (Of course, a full treatment of those fluctuations would require a complete theory of quantum gravity.) We will linearize and quantize those metric perturbations including their interaction with the quantum matter fields. This can be described more precisely in terms of $N$ identical matter fields. Our approach corresponds then to computing the quantum correlation functions for the metric perturbations to leading order in a $1 / N$ expansion.

In fact, one can show that the leading order contribution to the quantum correlation functions in a large $N$ expansion is equivalent to the stochastic correlation functions obtained in the context of stochastic semiclassical gravity. Whereas SCG is based on the semiclassical Einstein equation with sources given by the expectation value of the stress tensor operator of the quantum matter fields, stochastic semiclassical gravity is based on the Einstein-Langevin equation, which has in addition sources due to the noise kernel. The noise kernel is the symmetrized connected part of the twopoint quantum correlation function of the stress tensor operator with respect to the state of the matter fields and describes their stress-energy fluctuations.

Making use of the equivalence between quantum and sto- 
chastic correlation functions in stochastic semiclassical gravity, one is naturally led to separate the symmetrized quantum correlation function for the metric perturbations (to leading order in $1 / N$ ) into two separate contributions: intrinsic and induced fluctuations. The former are connected to the dispersion of the initial state of the metric perturbations, whereas the latter are proportional to the noise kernel and are induced by the quantum fluctuations of the matter field stress tensor operator.

b. Validity of semiclassical gravity. Different aspects concerning the validity of the description provided by SCG in the case of free quantum matter fields in the Minkowski vacuum state propagating on Minkowski spacetime have been studied by a number of authors. Most of them considered the stability of such a solution of SCG with respect to small perturbations of the metric. Horowitz was the first one to analyze the equations describing those perturbations, which involved higher order derivatives (up to fourth order), and found unstable solutions that grow exponentially with characteristic time scales comparable to the Planck time $[16,17]$. This was later reanalyzed by Jordan with similar conclusions [18]. However, those unstable solutions were regarded as an unphysical artifact by Simon, who argued that they lie beyond the expected domain of validity of the theory and emphasized that only those solutions which resulted from truncating perturbative expansions in terms of the square of the Planck length are acceptable [19,20]. Further discussion was provided by Flanagan and Wald [21], who advocated the use of an order reduction prescription first introduced by Parker and Simon [22] but insisted that even nonperturbative solutions of the resulting second order equation should be regarded as acceptable. Following these approaches Minkowski spacetime is shown to be a stable solution of SCG with respect to small metric perturbations.

Anderson, Molina-París and Mottola have recently taken up the issue of the validity of SCG [3] again. Their starting point is the fact that the semiclassical Einstein equation will fail to provide a valid description of the dynamics of the mean spacetime geometry whenever the higher order radiative corrections to the effective action, involving loops of gravitons or internal graviton propagators, become important (see Refs. [23-26] for some attempts to include those effects). Next, they argue qualitatively that such higher order radiative corrections cannot be neglected if the metric fluctuations grow without bound. Finally, they propose a criterion (a necessary condition) to characterize the growth of the metric fluctuations, and hence the validity of SCG, based on the stability of the solutions of the linearized semiclassical equation.

c. Our criterion. In this paper we address the issue of the stability of semiclassical solutions with respect to small quantum corrections. When the metric perturbations are quantized, the semiclassical equation can be interpreted as the equation governing the evolution of the expectation value of the operator for the metric perturbations. We introduce a stability criterion based on whether the metric fluctuations grow without bound or not by considering the behavior of the quantum correlation functions of the metric perturbations. Furthermore, we emphasize that one should consider not only the intrinsic fluctuations, but also the induced ones. In fact, the induced fluctuations play a crucial role when considering the stability of simple open quantum systems for several reasons. First, those systems usually exhibit a characteristic relaxation time so that for much larger times the contribution from the intrinsic fluctuations becomes negligible. Second, after that transient period the stability around an equilibrium configuration is the result of a balance between the energy dissipated by the system and the fluctuations induced by the environment, which is encoded in the so-called fluctuation-dissipation relation connecting the dissipation and the noise kernels.

It is true that the effect of intrinsic fluctuations can be deduced from an analysis of the solutions of the perturbed semiclassical Einstein equation, but in general one cannot retrieve the effect of the induced fluctuations from it. This effect can be properly accounted for in the stochastic semiclassical gravity framework. Both intrinsic and induced fluctuations are innate in the Einstein-Langevin equation.

d. Ford's program. Ford [4] was among the first to have noted the importance of quantum fluctuations in these issues. An earlier criterion put forth by Kuo and Ford [5] used the variance of the fluctuations of the stress tensor operator compared to the mean value as a measure of the validity of SCG. As pointed out by Hu and Phillips $[27,28]$ (see reply by Ford and $\mathrm{Wu}$ [7]) such a criterion should be refined by considering the back reaction of those fluctuations on the metric. Ford and collaborators also considered both intrinsic ("active") [9-11] and induced ("passive") [4-8] fluctuations, but they did not treat them in a unified way and did not discuss their precise relation to the quantum correlation function for the metric perturbations. Furthermore, they did not include the full averaged back reaction of the matter fields selfconsistently, and the contributions from vacuum fluctuations in Minkowski space were discarded. As these issues have been discussed before by both groups of Ford and $\mathrm{Hu}$, we will only make a few remarks at the end of this paper.

Here, our attention will be focused on comparing the criteria based on the linear response approach proposed by Anderson et al. and our stochastic gravity approach. Since the differences in the two ways to address the issue of the validity of SCG are rooted in the difference between linear response theory and stochastic dynamics as applied to SCG, we hope that this work can also serve the purpose of offering a comparison between these two important approaches exploring the validity of the mean field approximation. In the examples provided, we will specialize the matter fields to the case of free scalar fields, but generalization to vectorial or fermionic fields should not pose major difficulty.

e. Terminology and organization. To avoid unnecessary ambiguities or confusion in interpretation, it is useful to clarify the use of some terminology here.

First, a comment on the difference between the stochastic gravity program in general and its present implementation status in particular. Stochastic semiclassical gravity can be understood as the Gaussian approximation to stochastic gravity. Although technically the actual implementations of stochastic gravity so far (to which our present discussion applies) have been restricted to linear metric perturbations 
around the background geometry and a Gaussian stochastic source, the theoretical construct of the stochastic gravity program has a much broader meaning beyond these limitations. It refers to the range of theories based on second and higher order correlation functions. Noise can be defined in fully nonlinear theories (e.g. correlation noise [29] in the Schwinger-Dyson equation hierarchy) to some degree, ${ }^{1}$ but one should not expect the simple Langevin form with Gaussian and additive noise to prevail. Thus, stochastic gravity in this broad sense entails the whole hierarchy of correlation functions, which would imply going beyond order $1 / N$ in the generating functional. It could in principle provide the means [similar to the Bogoliubov-Born-Green-Kirkwood-Yvon (BBGKY) hierarchy in kinetic theory] to access the full theory of quantum gravity $[14,15]$. It is in this sense that we say stochastic gravity is the intermediate theory between SCG (a mean field theory based on the expectation value of the energy momentum tensor of quantum fields) and quantum gravity (understood as the full hierarchy of correlation functions retaining complete quantum coherence).

Second, the precise meaning in our use of the terms perturbations and fluctuations. By perturbations of the metric we mean deviations of the perturbed metric from a background metric. Perturbations are purely classical and deterministic in general relativity and SCG. In stochastic gravity, they are classical but stochastic (with a vanishing statistical expectation value) so that the background configuration can be regarded as the expectation value of a stochastic metric (a complete gauge fixing is required to meaningfully talk about the expectation value of a metric). In linear quantum gravity, perturbations are quantum operators. For a state with a vanishing expectation value, the background metric can then be regarded as the expectation value of the metric operator (again a complete gauge fixing is required) times the identity operator. On the other hand, the term fluctuations is employed only to refer to the statistical fluctuations of the metric perturbations when they correspond to a stochastic process or to the quantum fluctuations of the metric perturbations when they are treated as a quantum operator.

Third, by leading order in the large $N$ limit we mean the lowest order in $1 / N$ with a nonvanishing contribution. Hence, as we will see, the leading order for the source of the semiclassical Einstein equation, which is proportional to the expectation value of the stress tensor operator, is $1 / N^{0}$, whereas the leading order for the quantum two-point correlation functions is $1 / N$.

The paper is organized as follows. In Sec. II we briefly review the fundamental aspects of SCG and how one can study linearized perturbations around a background solution of SCG. This is generalized to incorporate the metric fluctuations in Sec. III, where the key elements of stochastic semiclassical gravity are introduced and the equivalence between stochastic and quantum correlation functions is explained. In Sec. IV we propose a generalized stability crite-

\footnotetext{
${ }^{1}$ In general, it might be necessary to extend the concept of stochastic process to that of processes with a real and normalized distribution functional but not necessarily positive definite.
}

rion that includes the metric fluctuations, which is then applied to the specific case of a Minkowski background. We conclude by summarizing and discussing the main results in Sec. V.

A number of additional details and technical points are left for the Appendixes. In Appendix A we illustrate the basic aspects of intrinsic and induced fluctuations using a simple quantum Brownian motion model. In Appendix B we provide the expressions for the dissipation and noise kernels in a Minkowski spacetime and the vacuum state. Some of the main steps to show the equivalence between stochastic and correlation functions using a large $N$ expansion are summarized in Appendix C. The physical interpretation of the singular coincidence limit for the noise kernel and possible ways to deal with it are explained in Appendix D. Finally, in Appendix E we discuss the existence of runaway solutions in SCG and stochastic semiclassical gravity as well as methods to deal with them.

Throughout the paper we use natural units with $\hbar=c=1$ and the $(+,+,+)$ convention of Ref. [30]. We also make use of the abstract index notation of Ref. [31]. Latin indices denote abstract indices, whereas Greek indices are employed when a particular coordinate system is considered.

\section{SEMICLASSICAL GRAVITY AND LINEAR RESPONSE THEORY}

A possible first step when addressing the interplay between gravity and quantum field theory is to consider the evolution of quantum matter fields (matter field is referred to here as any field other than the gravitational one) on a classical spacetime with a nontrivial geometry, characterized by a metric $g_{a b}$. As opposed to the situation for a Minkowski spacetime, there is in general no preferred vacuum state for the fields and particle creation effects naturally arise, such as Hawking radiation for black holes, cosmological particle creation and the generation of primordial inhomogeneities in inflationary cosmological models. Quantum field theory in curved spacetimes (QFTCST) is by now a well-established subject (at least for free fields and globally hyperbolic spacetimes) [32,33].

QFTCST is only an approximation in that the matter fields are treated as test field evolving on a given spacetime. Einstein's theory requires the spacetime dynamics to determine and be determined by the matter fields. Thus one needs to consider the back reaction of the quantum matter fields on the dynamics of the spacetime geometry, which naturally leads to the semiclassical theory of gravity, where the evolution of the spacetime metric $g_{a b}$ is determined by the semiclassical Einstein equation

$$
G_{a b}[g]+\Lambda g_{a b}-\alpha A_{a b}[g]-\beta B_{a b}[g]=\kappa\left\langle\hat{T}_{a b}[g]\right\rangle_{\text {ren }}^{\prime},
$$

where $g_{a b}$ is the spacetime metric, $G_{a b}[g]$ is the Einstein tensor and the matter source corresponds to the renormalized expectation value of the stress tensor operator of the matter fields (a prime was used to distinguish it from that introduced below after absorbing some terms). Here, $\Lambda$ is the 
renormalized cosmological constant and $\kappa=8 \pi G$, with $G$ $\equiv 1 / m_{p}^{2}$ being the Newton constant and $m_{p}$ the Planck mass; $\alpha$ and $\beta$ are renormalized dimensionless coupling constants associated with tensors $A_{a b}[g], B_{a b}[g]$ needed for the renormalization of the logarithmic divergences. ${ }^{2}$ The expectation value of the stress tensor operator exhibits divergences which are local and state independent. Introducing a covariant regularization and renormalization procedure, those divergences can be absorbed into the cosmological constant, the Newton constant multiplying the Einstein-Hilbert term and the gravitational action counterterms quadratic in the curvature. The finite contributions from those counterterms give rise to the covariantly conserved tensors $A_{a b}$ and $B_{a b}$ which result from functionally differentiating with respect to the metric the terms $\int d^{4} x \sqrt{-g} C^{a b c d} C_{a b c d}$ and $\int d^{4} x \sqrt{-g} R^{2}$ respectively, where $C_{a b c d}$ is the Weyl tensor and $R$ is the Ricci scalar. Those contributions were explicitly written on the left-hand side of Eq. (1), but from now on will be included in the renormalized expectation value of the stress tensor operator so that the semiclassical Einstein equation becomes

$$
G_{a b}[g]=\kappa\left\langle\hat{T}_{a b}[g]\right\rangle_{\text {ren }} .
$$

The field operators appearing in the stress tensor operator for the quantum matter fields are in the Heisenberg picture and satisfy the corresponding equation of motion, which coincides with the classical field equation for fields evolving on that spacetime. In particular, if we consider a free scalar field, the field operator in the Heisenberg picture will satisfy the corresponding Klein-Gordon equation for that geometry.

Given a manifold $\mathcal{M}$ and a metric $g_{a b}$ which characterize a globally hyperbolic spacetime, and a density matrix $\hat{\rho}$ which specifies the state of the quantum matter fields on a particular Cauchy hypersurface, the triplet $\left(\mathcal{M}, g_{a b}, \hat{\rho}\right)$ constitutes a solution of SCG if it is a self-consistent solution of both the semiclassical Einstein equation (2) and the equations of motion for the quantum operators of the matter fields evolving on the spacetime manifold $\mathcal{M}$ with metric $g_{a b}$. Those operators enter in turn into the definition of the stress tensor operator appearing in the semiclassical Einstein equation.

The semiclassical Einstein equation has been justified in at least two different ways. One possibility is to argue, by assuming a number of reasonable axioms, that it is the only consistent way to extend the classical Einstein equation to couple the quantum matter fields to a classical metric $[21,33]$. Alternatively, it can be derived by considering $N$ free matter fields weakly coupled to the gravitational field in the sense that the gravitational coupling constant times the number of fields $N G$ remains constant in the limit $N \rightarrow \infty$ [34]. The semiclassical Einstein equation can then be shown to

\footnotetext{
${ }^{2}$ The renormalized coupling constants are running coupling constants which depend on some renormalization scale $\mu$. However, since $\left\langle\hat{T}_{a b}[g]\right\rangle_{\text {ren }}^{\prime}$ has the same dependence on $\mu$, the semiclassical Einstein equation is invariant under the renormalization group, which involves changes in the renormalization scale $\mu$.
}

correspond to the dynamical equation for the evolution of the expectation value of the metric in the limit of large $N .^{3}$ Of course, in reality $N$ is finite and the semiclassical Einstein equation can only be understood as the lowest order contribution in a $1 / N$ expansion.

Functional methods based on path integrals are useful not only in the rendition of ideas but also in actual computations. However, the usual in-out formalism suitable for computing transition matrix elements in scattering problems is not appropriate to deal with back-reaction problems in which one is interested in the causal evolution of true expectation values from their initial values. The closed-time-path (CTP) formalism, which naturally yields true expectation values and causal evolution equations for their dynamics [35-39], should be used instead. This formalism has been applied to study a number of situations involving gravitational backreaction effects of quantum fields [40-44]. (See also Ref. [45] for an interesting application to an analogous situation in QED, where the back reaction of charged quantum fields on the dynamics of the expectation value of the electromagnetic field was considered.) This includes minisuperspace models which restrict the possible geometries to RobertsonWalker metrics and consider perturbative deviations from the massless conformal case for the matter fields [42-44], as well as small metric perturbations (of a less restricted form) around self-consistent solutions of SCG [40,41].

More specifically, given a background metric $g_{a b}$ which is a solution of the semiclassical Einstein equation in SCG, one can compute the CTP effective action on the perturbed metric $\tilde{g}_{a b}=g_{a b}+h_{a b}$ up to quadratic order in the metric perturbations $h_{a b}$. Taking the functional derivative of the CTP effective action with respect to $h_{a b}$, one gets the perturbed version of Eq. (2) to linear order in the metric perturbations:

$$
G_{a b}^{(1)}[g+h]=\kappa\left\langle\hat{T}_{a b}^{(1)}[g+h]\right\rangle_{\mathrm{ren}},
$$

where the superindex (1) was used to denote that only terms linear in $h_{a b}$ should be considered. The linearized Einstein tensor $G_{a b}^{(1)}[g+h]$ comes from the Einstein-Hilbert term in the gravitational action. On the other hand, the contribution to the CTP effective action which results from functionally integrating the matter fields and involves the expectation value

\footnotetext{
${ }^{3}$ One could be concerned that such a derivation was purely formal due to the impossibility of having a well-defined expectation value for the metric (at least without a complete gauge fixing), the difficulties in defining a measure for the path integral free of problems related to the gauge freedom under diffeomorphisms, and the issues related to the nonrenormalizable character of perturbative gravity. Nevertheless, in the limit $N \rightarrow \infty$ the contribution from the graviton loops vanishes so that the last two problems become irrelevant, whereas the fluctuations of the metric around a given background are completely suppressed and, hence, the first problem also disappears.
} 
and two-point correlation functions of the stress tensor operator on the background geometry yields the following result for the linearized expectation value of the stress tensor operator:

$$
\left\langle\hat{T}^{(1) a b}[g+h ; x)\right\rangle=-2(H \cdot h)^{a b}(x)-2(M \cdot h)^{a b}(x),
$$

where we have introduced the notation $A \cdot B$ $\equiv \int d^{4} y \sqrt{-g(y)} A^{a b}(y) B_{a b}(y)$, and the kernels $H$ and $M$ are given by

$$
\begin{aligned}
H^{a b c d}(x, y)= & -\frac{1}{4} \operatorname{Im}\left\langle T^{*} \hat{T}^{a b}[\hat{\varphi}, g ; x) \hat{T}^{c d}[\hat{\varphi}, g ; y)\right\rangle \\
& +\frac{i}{8}\left\langle\left[\hat{T}^{a b}[\hat{\varphi}, g ; x), \hat{T}^{c d}[\hat{\varphi}, g ; y)\right]\right\rangle, \\
M^{a b c d}(x, y)= & -\frac{1}{2}\left(\frac{1}{\sqrt{-g(x)}} \frac{\delta\left(\left\langle\hat{T}^{a b}\left[\hat{\varphi}, g_{a b} ; x\right)\right\rangle\right)}{\delta g_{c d}(y)}\right),
\end{aligned}
$$

where the notation $T^{*}$ was employed to indicate that the spacetime partial derivatives appearing in the time-ordered operators also act on the theta function implementing the time ordering. The functional derivative appearing on the right-hand side of Eq. (6) should be understood to account only for the explicit dependence on the metric: the implicit dependence through the field operator $\hat{\varphi}[g]$ is excluded. ${ }^{4}$

The previous result for the expectation value of the stress tensor when small metric perturbations around a background solution of SCG are considered has been obtained in two ways: (1) by applying the usual techniques of linear response theory (see, for instance, [46]) to SCG $[3,47,48]$ and (2) by applying the influence functional formalism $[49,50]$ to linearized metric perturbations regarded as an open quantum system interacting with an environment constituted by the quantum matter fields [51,52]. The influence functional approach also provides the noise kernel which underscores the stochastic nature of the dynamics for the metric perturbations. We will employ this method in the next section.

The explicit expression for the linearized expectation value $\left\langle\hat{T}^{(1) a b}[g+h ; x)\right\rangle$ in the particular case of a Minkowski background spacetime and a free scalar field in the Minkowski vacuum state was obtained in Refs. [16] and [21] for a massless field and in Refs. [3,53,54] for a field with an

\footnotetext{
${ }^{4}$ The kernels $H$ and $M$ both exhibit divergences, but they can be removed by the standard procedure for renormalizing the expectation value of the stress tensor in an arbitrary spacetime (after all they are related to the terms in $\left\langle\hat{T}^{a b}[g+h ; x)\right\rangle$ that are linear in $h_{a b}$ ), which involves renormalizing the cosmological constant and the Newton constant in the bare gravitational action as well as including counterterms quadratic in the curvature. More precisely, by evaluating all the counterterms in the bare gravitational action on the perturbed metric and keeping the terms quadratic in the metric perturbations, which give rise to linear terms in $\left\langle\hat{T}^{a b}[g ; x)\right\rangle$, the divergences in the CTP effective action arising from the kernels $H$ and $M$ are exactly canceled.
}

arbitrary mass. For our discussion we have included them in Appendix B, where a global inertial coordinate system $\left\{x^{\mu}\right\}$ for the Minkowski background is used. According to Eq. (B6), $\left\langle\hat{T}^{(1) a b}[g+h]\right\rangle_{\text {ren }}$ can be written entirely in terms of the linearized Einstein tensor $G^{(1) a b}$. Taking that into account, the expression for the linearized semiclassical Einstein equation (3) in Fourier space becomes

$$
F_{\alpha \beta}^{\mu \nu}(p) \widetilde{G}^{(1) \alpha \beta}(p)=0,
$$

where

$$
F_{\alpha \beta}^{\mu \nu}(p)=F_{1}(p) \delta_{(\alpha}^{\mu} \delta_{\beta)}^{\nu}+F_{2}(p) p^{2} P^{\mu \nu} \eta_{\alpha \beta},
$$

with

$$
F_{1}(p)=1+2 \kappa p^{2}\left[\widetilde{H}_{\mathrm{A}}(p)-2 \bar{\alpha}\right]
$$

$$
F_{2}(p)=-\frac{2 \kappa}{3}\left[\widetilde{H}_{\mathrm{A}}(p)+3 \widetilde{H}_{\mathrm{B}}(p)-2 \bar{\alpha}-6 \bar{\beta}\right]
$$

where $\bar{\alpha}$ and $\bar{\beta}$ are some constants which include the renormalized parameters $\alpha$ and $\beta$ in Eq. (1), and the kernels $\widetilde{H}_{A}(p)$ and $\widetilde{H}_{B}(p)$ are defined in Eqs. (B3) and (B4) of Appendix B. The solutions of Eq. (7) were analyzed in Refs. [16] and [21] for the massless case and Ref. [3] for the general case. There is an obvious solution for $\widetilde{G}_{a b}^{(1)}(p)=0$, which corresponds to linear gravitational waves propagating in Minkowski spacetime. In addition, there are solutions of the form $\widetilde{G}_{\mu \nu}^{(1)} \propto \delta\left(p^{2}-p_{0}^{2}\right.$ ) for particular values of $p_{0}^{2}$ (positive or negative) comparable to $m_{p}^{2}$. Since they exhibit characteristic time scales or length scales comparable to the Planck scale, where semiclassical gravity is not expected to be reliable anymore, they are usually regarded as unphysical. A more detailed discussion of this kind of solutions is given in Sec. IV and Appendix E.

In fact, as will be explained in Sec. IV, if one quantizes the linearized metric perturbations, Eq. (7) coincides with the equation governing the evolution of the expectation value of the operator $\hat{h}_{a b}$ for the metric perturbations. Therefore, an analysis of the stability of the solutions of linearized semiclassical Einstein equation (7) can be equivalently understood in terms of the evolution for the expectation value of $\hat{h}_{a b}$. In Sec. IV we will argue that a stability analysis for solutions of SCG with respect to small quantum corrections based solely on the expectation value of the metric perturbations is incomplete and should be extended to take into account the metric fluctuations as well. Stochastic semiclassical gravity is particularly well suited to study the fluctuations of the metric and will play an important role in our later discussions. Therefore, in the next section we briefly review the formalism based on the Einstein-Langevin equation for small metric perturbations around semiclassical solutions within the framework of stochastic semiclassical gravity. 


\section{EINSTEIN-LANGEVIN EQUATION FOR METRIC PERTURBATIONS AROUND A GIVEN BACKGROUND}

The semiclassical Einstein equation, which takes into account only the mean values, is inadequate whenever the fluctuations of the stress tensor operator are important. An improved treatment is provided by the Einstein-Langevin equation of stochastic gravity, which contains a (Gaussian) stochastic source with a vanishing expectation value and a correlation function characterized by the symmetrized twopoint function of the stress tensor operator. This theory has been discussed by a number of authors $[1,2,42-44,51,55,56]$. Consider a globally hyperbolic background spacetime and an initial state for the quantum matter fields (one usually restricts to free fields) which is a self-consistent solution of SCG; i.e., it satisfies the semiclassical Einstein equation with the expectation value of the stress tensor operator obtained by considering the evolution of the matter fields on the same background geometry. The Einstein-Langevin equation governing the dynamics of the linearized perturbations $h_{a b}$ around the background metric $g_{a b}$ is given by

$$
G_{a b}^{(1)}[g+h]=\kappa\left\langle\hat{T}_{a b}^{(1)}[g+h]\right\rangle_{\mathrm{ren}}+\kappa \xi_{a b}[g],
$$

where the Gaussian stochastic source $\xi_{a b}[g]$ is completely characterized by its correlation function in terms of the noise kernel $\mathcal{N}_{a b c d}(x, y)$, which accounts for the fluctuations of the stress tensor operator, as follows:

$$
\begin{aligned}
\left\langle\xi_{a b}[g ; x) \xi_{c d}[g ; y)\right\rangle_{\xi} & =\mathcal{N}_{a b c d}(x, y) \\
& \equiv \frac{1}{2}\left\langle\left\{\hat{t}_{a b}[g ; x), \hat{t}_{c d}[g ; y)\right\}\right\rangle,
\end{aligned}
$$

where $\hat{t}_{a b} \equiv \hat{T}_{a b}-\left\langle\hat{T}_{a b}\right\rangle$ and $\langle\cdots\rangle$ is the usual expectation value with respect to the quantum state of the matter fields, whereas $\langle\cdots\rangle_{\xi}$ denotes taking the average with respect to all possible realizations of the stochastic source $\xi_{a b}$. Note that any local term quadratic in the curvature arising from finite contributions of the counterterms required to renormalize the bare expectation value of the stress tensor operator has been absorbed into its renormalized version $\left\langle\hat{T}_{a b}^{(1)}[g+h]\right\rangle_{\text {ren }}$. It should also be emphasized that solutions of the EinsteinLangevin equation for the metric perturbations are classical stochastic tensorial fields, not quantum operators.

The precise meaning that should be given to these stochastic metric perturbations and the relation of the corresponding stochastic correlation functions to the quantum fluctuations resulting from quantizing these metric perturbations will be discussed below. Before doing so, it is, however, useful to mention some of the basic properties of the Einstein-Langevin equation (a more detailed discussion can be found in Refs. [1,2,51]). First, when taking the average of the Einstein-Langevin equation (11) with respect to all the possible realizations of the stochastic source, we recover the semiclassical Einstein equation (3), as follows straightforwardly from the vanishing expectation value of the stochastic source. Second, the integrability of the Einstein-Langevin equation is guaranteed, in the same way as in the semiclassical Einstein equation, by conservation of the matter sources. Conservation of the expectation value of the stress tensor operator follows immediately from the fact that the divergence (with respect to the covariant derivative) of the stress tensor operator vanishes when the equation of motion of the matter field operators is satisfied (e.g. the KleinGordon equation for a scalar field). On the other hand, the fact that $\left\langle\nabla^{a} \xi_{a b}(x)\right\rangle_{\xi}=0$ and $\left\langle\xi_{a b}(x) \nabla^{c} \xi_{c d}(x)\right\rangle_{\xi}=0$, which completely characterize the Gaussian stochastic field $\nabla^{a} \xi_{a b}(x)$, guarantees the conservation of the stochastic source (unless otherwise stated, from now on all the covariant derivatives are taken in the background metric and indices are raised and lowered using also the background metric). The previous two equalities are, respectively, a consequence of the vanishing expectation value of the stochastic source and the fact that $\nabla^{a} \hat{t}_{a b}(x)=0$. Finally, the Einstein-Langevin equation is invariant under gauge transformations corresponding to infinitesimal diffeomorphisms characterized by an arbitrary vector field $\vec{\zeta}(x)$, which generates transformations for the metric of the form $h_{a b} \rightarrow h_{a b}$ $+\nabla_{a} \zeta_{b}+\nabla_{b} \zeta_{a}$. This fact can be seen by realizing that the stochastic source does not depend on the metric perturbations, whereas the terms depending on the metric perturbations are all together gauge invariant. This is because they correspond to perturbing the semiclassical Einstein equation for the background metric $g_{a b}$, which is automatically satisfied since the background configuration under consideration for the metric and the state of the matter fields is a solution of SCG.

The Einstein-Langevin equation had been previously derived making use of a formal analogy with open quantum systems and employing the influence functional formalism $[49,50]$. This form was also justified in Ref. [57] by arguing that it is the only consistent generalization of the semiclassical Einstein equation which takes into account the lowest order effects due to the fluctuations of the stress tensor operator. In fact, making use of a large $N$ expansion, one can show that the stochastic correlation functions for the metric perturbations obtained from the Einstein-Langevin equation coincide with the leading order contribution to the quantum correlation functions in the large $N$ limit. The details of the derivation will be given in Ref. [58] and are summarized in Appendix $\mathrm{C}$ for the particular case of a Minkowski background, to which we will restrict in the present discussion. In particular, the two-point stochastic correlation function is equivalent to the symmetrized quantum correlation function to leading order in $1 / N$ provided that one also averages over the initial conditions for the solutions of the EinsteinLangevin equation distributed according to the Wigner functional characterizing the initial state of the metric perturbations [see Eq. (C11) in Appendix C for the definition of the Wigner functional]. It is, therefore, convenient to express the solutions of the Einstein-Langevin equation as

$$
h_{a b}(x)=\Sigma_{a b}^{(0)}(x)+\bar{\kappa}\left(G_{\mathrm{ret}} \cdot \xi\right)_{a b}(x),
$$

where $\bar{\kappa}=N \kappa$ is the rescaled gravitational coupling constant introduced in Appendix C, $\Sigma_{a b}^{(0)}(x)$ is a solution of the homogeneous part of the Einstein-Langevin equation (11) con- 
taining all the information about the initial conditions [by homogeneous part we mean Eq. (11) excluding the stochastic source, which coincides with the semiclassical Einstein equation (2)], and $G_{\text {ret }}\left(x, x^{\prime}\right)$ is the retarded propagator with vanishing initial conditions associated with that equation (see Appendix E 3 for important remarks on the propagator). Using Eq. (12), we can then get the following result for the symmetrized two-point quantum correlation function:

$$
\begin{aligned}
\frac{1}{2}\left\langle\left\{\hat{h}_{a b}(x), \hat{h}_{c d}\left(x^{\prime}\right)\right\}\right\rangle= & \left\langle\Sigma_{a b}^{(0)}(x) \Sigma_{c d}^{(0)}\left(x^{\prime}\right)\right\rangle_{\Sigma_{a b}^{(i)}, \Pi_{(i)}^{c d}} \\
& +\frac{\bar{\kappa}^{2}}{N}\left(G_{\mathrm{ret}} \cdot \mathcal{N} \cdot\left(G_{\mathrm{ret}}\right)^{T}\right)_{a b c d}\left(x, x^{\prime}\right),
\end{aligned}
$$

where the Lorentz gauge condition $\nabla^{a}\left(\bar{h}_{a b}-1 / 2 \eta_{a b} h_{c}^{c}\right)=0$ as well as some initial condition to fix completely the remaining gauge freedom of the initial state should be implicitly understood, and the stochastic source was rescaled according to Appendix $\mathrm{C}$ so that $\left\langle\xi_{a b}[g ; x) \xi_{c d}[g ; y)\right\rangle_{\xi}$ $=(1 / N) \mathcal{N}_{a b c d}(x, y)$, where $\mathcal{N}_{a b c d}(x, y)$ is the noise kernel for a single field.

This result is analogous to that obtained in Ref. [59] for linear QBM models and briefly summarized in Appendix A. It should be emphasized that, similar to that case, there are two different contributions to the symmetrized quantum correlation function. The first one is connected to the quantum fluctuations of the initial state of the metric perturbations and we will refer to it as intrinsic fluctuations. The second contribution, proportional to the noise kernel, accounts for the fluctuations due to the interaction with the matter fields, and we will refer to it as induced fluctuations. In the next section we will formulate a generalized stability criterion for the solutions of SCG which involves the quantum fluctuations of the metric. In particular we will see that the induced fluctuations will play an important role on that issue.

The noise kernel that we need for our discussions is for the particular case of a Minkowski background spacetime with a scalar field in the Minkowski vacuum. It was obtained in Ref. [54] and is given by Eq. (B7) in Appendix B.

\section{STABILITY CRITERION FOR SOLUTIONS OF SEMICLASSICAL GRAVITY}

In this section we will propose a criterion for analyzing the stability of a given solution of SCG with respect to small quantum corrections, associated with quantized metric perturbations around a background geometry. As an important example, we will apply this to the particular case of a Minkowski background with $N$ scalar fields in the Minkowski vacuum state.

\section{A. Stability of Minkowski space: Previous criteria}

The stability of metric perturbations around a Minkowski spacetime interacting with quantum matter fields in their Minkowski vacuum state was first studied in the context of SCG by Horowitz [16]. He considered massless conformally coupled scalar fields and found exponential instabilities for the linearized metric perturbations with characteristic time scales comparable to the Planck time. Those solutions are closely related to the higher derivative countertems required to renormalize the expectation value of the stress tensor operator (see, however, Appendix E for further comments on this point) and are analogous to the runaway solutions commonly present in radiation reaction processes such as those considered in classical electrodynamics $[60,61]$. It is generally believed that the runaway solutions obtained by Horowitz are an unphysical artifact since they involve scales beyond the regime where SCG is expected to be reliable (in fact, this statement can be naturally formulated when regarding general relativity as a low energy effective theory).

Since the existence of terms with higher derivatives in time implies an increase in the number of degrees of freedom (in an initial value formulation, not only the metric and its time derivative should be specified, but also its second and third order time derivatives), it seems plausible that, by restricting to an appropriate subspace of solutions of the semiclassical Einstein equation, one can reestablish the usual number of degrees of freedom in general relativity and, at the same time, get rid of all the unphysical runaway solutions. Following this line of thought Simon proposed that one should restrict to solutions which result from truncating to order $\hbar$ an analytic expansion in $\hbar$ (or equivalently in $l_{p}^{2}$, the Planck length squared) $[19,20]$. Together with Parker he also introduced a prescription to reduce the order of the semiclassical Einstein equation which was computationally convenient in order to obtain solutions corresponding to such truncated perturbative expansions in $\hbar$ [22].

On the other hand, Flanagan and Wald argued that Simon's criterion based on truncating to order $\hbar$ solutions which correspond to analytic expansions in $\hbar$ seemed too restrictive since it only allowed small deviations with respect to the classical solutions of the Einstein equations [21]. In particular, one would miss those situations in which the small semiclassical corrections build up to give significant deviations at long times, such as those corresponding to the evaporation of a macroscopic black hole (with a mass much larger than the Planck mass) by emission of Hawking radiation. Furthermore, they illustrated with simple examples that there are cases in which one expects that no solutions of the semiclassical equation are analytic in $\hbar$. Therefore, they suggested that, rather than trying to restrict the subspace of acceptable solutions, one should simply transform the semiclassical equation, by making use of Simon and Parker's order reduction prescription, to a second order equation which were equivalent to the original equation up to the order in $\hbar$ (or $l_{p}^{2}$ ) under consideration. All the solutions of the second order equation should then be regarded as acceptable, even if they are not analytic in $\hbar$. Obviously, one could only extract physically reliable information from those solutions for scales much larger than the Planck length.

Yet another prescription was proposed by Anderson, Molina-París and Mottola [3] on the stability of small metric perturbations around the Minkowski spacetime. They got rid 
of the unphysical runaway solutions by working in Fourier space and discarding those solutions which corresponded to 4-momenta with modulus comparable or larger in absolute value than the Planck mass. However, it is not clear how this procedure could be generalized to situations where working in Fourier space is not adequate, as in time-dependent background spacetimes.

The consequences of both the order reduction prescription introduced by Simon and Parker and advocated by Flanagan and Wald and the procedure employed by Anderson et al. are rather drastic, at least when applied to the case of a Minkowski background, since one is just left with the solutions of the sourceless classical Einstein equation corresponding to linear gravitational waves propagating in Minkowski spacetime. In fact, the situation was not completely trivial for Flanagan and Wald, who were interested in analyzing whether the averaged null energy condition (ANEC) was satisfied in SCG by considering perturbations of the Minkowski solution, because they also perturbed the state of the matter fields. The order reduction prescription also seems to exclude those solutions which correspond to inflationary models driven entirely by the vacuum polarization of the quantum matter fields [62], such as the trace anomaly driven inflationary model initially proposed by Starobinsky [63]. To keep this kind of models, Hawking, Hertog and Reall considered a less drastic alternative to deal with the runaway solutions $[64,65]$. Their procedure, which is analogous to some methods previously employed in classical electrodynamics for radiation reaction problems [60], is based on discarding solutions which grow without bound at late times (see Appendix $\mathrm{E}$ for further discussions on this and related issues).

\section{B. Generalized stability criterion}

How does one characterize the quantum state of the metric perturbations? The first candidate is the expectation value for the operator associated with the perturbation of the metric, $\hat{h}_{a b}$. In fact, using a large $N$ expansion, Hartle and Horowitz showed that the semiclassical Einstein equation can be interpreted as the equation governing the evolution of the expectation value of the metric to leading order in $1 / N$ [34]. Taking that result into account, the study of the stability of a solution of SCG by linearizing the semiclassical Einstein equation with respect to small metric perturbations around that solution can be understood in the following way: Take an initial state for the metric perturbations with a small nonvanishing expectation value for the operator $\hat{h}_{a b}$, let it evolve, and see if the expectation value grows without bound.

However, in addition to the expectation value of $\hat{h}_{a b}$ the state of the metric perturbations will also be characterized by its fluctuations. In fact, if there was no interaction with matter fields so that the state for the metric perturbations evolved unitarily, the set of quantum correlation functions (for the operator $\hat{h}_{a b}$ ) evaluated at equal times would completely characterize the quantum state of the metric perturbations. ${ }^{5}$ Let us now suppose that the evolution of the expectation value is stable (i.e. that it does not grow unboundedly with time) or even that it vanishes for all times. It is clear that the semiclassical solution cannot be regarded as stable with respect to small quantum corrections if the fluctuations of the state for the metric perturbations grow without bound. Therefore, the stability criterion stated in Ref. [3] should be generalized: one also needs to take into account the fluctuations. According to Ref. [3], a necessary condition for the stability of a solution of SCG requires that no gauge invariant scalar quantity constructed just from the linearized metric perturbation $h_{a b}$ (which satisfies the semiclassical Einstein equation linearized around the semiclassical solution under consideration) and its derivatives grows without bound. This criterion can be interpreted as a condition on the stability of the expectation value of the operator $\hat{h}_{a b}$ for the state of the metric perturbations. We claim that, in addition, the $n$-point quantum correlation functions for the metric perturbations (starting with $n=2$ ) should also be stable. Considerations based on gauge-invariant variables will not be necessary because we will be dealing with expressions where the gauge freedom has been completely fixed.

As explained in Appendix C, to leading order in $1 / N$ the CTP generating functional for the metric perturbations exhibits a Gaussian form provided that a Gaussian initial state for the metric perturbations with vanishing expectation value is chosen. All the $n$-point quantum correlation functions can then be obtained, to leading order in $1 / N$, from the two-point quantum correlation function. Furthermore, any of the twopoint quantum correlation functions can in turn be expressed in terms of the symmetrized and antisymmetrized correlation functions (the expectation values of the commutator and anticommutator of the operator $\hat{h}_{a b}$ ). To leading order in $1 / N$ the commutator is independent of the initial state of the metric perturbations and is given by $2 i \kappa\left[G_{\text {ret }}\left(x^{\prime}, x\right)\right.$ $\left.-G_{\mathrm{ret}}\left(x, x^{\prime}\right)\right]$. On the other hand, the expectation value of the anticommutator is given by Eq. (14) and is the sum of two separate contributions: intrinsic and induced fluctuations.

The first contribution in Eq. (14) to the correlation function for the metric perturbations involves the solutions of the homogeneous part of the Einstein-Langevin equation (11), which actually coincides with the linearized semiclassical equation for the metric perturbations around the background geometry. Similarly, $G_{\text {ret }}$ corresponds to the retarded propagator (with vanishing initial conditions) associated with the

\footnotetext{
${ }^{5}$ Nevertheless, since the metric perturbations constitute an open quantum system due to the interaction with the matter fields, their state should be described by a density matrix (the reduced density matrix obtained by taking the density matrix for the whole system-metric perturbations plus matter fields-and tracing out the matter fields) which exhibits a nonunitary and even nonMarkovian evolution. Therefore, as explained in Ref. [59], the correlation functions involving different times may contain information which cannot be obtained just from the correlation functions evaluated at equal times.
} 
linearized semiclassical equation. Thus, solving the perturbed semiclassical Einstein equation not only accounts for the evolution of the expectation value of the metric perturbations, which will exhibit a nontrivial dynamics as long as we choose an initial state with nonvanishing expectation value, but also provides nontrivial information, even for a state with a vanishing expectation value, about the commutator as well as the intrinsic fluctuations of the metric. This implies that the analysis about the stability of the solutions of SCG can also be used to determine the stability of the metric perturbations with respect to intrinsic fluctuations.

The new observation we make here is that the induced fluctuations can be important as well. Both the retarded propagator and the solutions of the linearized semiclassical Einstein equation depend, through the kernel $H$, on the expectation value of the commutator of the stress tensor operator on the background geometry and on the imaginary part of its time-ordered two-point function. However, they do not involve the expectation value of the anticommutator, which drives the induced fluctuations. Furthermore, although the expectation values of the commutator and anticommutator are related by a fluctuation-dissipation relation in some particular cases [51,54], that is not true in general and the induced fluctuations need to be explicitly analyzed.

To sum up, when analyzing the stability of a solution of SCG with respect to small quantum corrections, one should also consider the behavior of both the intrinsic and induced fluctuations of the quantized metric perturbations. Whereas information on the stability of the intrinsic fluctuations can be retrieved from an analysis of the solutions of the perturbed semiclassical Einstein equation, the effect of the induced fluctuations is properly accounted for only in the stochastic semiclassical gravity framework based on the Einstein-Langevin equation.

\section{Stability of Minkowski space from our criterion}

We now turn to the application of the criterion proposed in the previous subsection to the particular yet important case of Minkowski spacetime. As explained there, the existing results in the literature can be interpreted as analysis of the stability of the expectation value of the operator associated with the metric perturbations (see, however, Refs. $[17,34,66])$. On the other hand, we also need to include in our consideration the fluctuations, characterized by the twopoint quantum correlation function.

Before proceeding to analyze the two-point quantum correlation functions it is convenient to decompose the metric perturbations around Minkowski spacetime in the following way [3]:

$$
h_{a b}=\phi \eta_{a b}+\left(\nabla_{(a} \nabla_{b)}-\eta_{a b} \square\right) \psi+2 \nabla_{(a} v_{b)}+h_{a b}^{\mathrm{TT}},
$$

where $v^{a}$ is a transverse vector and $h_{a b}^{\mathrm{TT}}$ is a transverse and traceless symmetric tensor, i.e. $\nabla_{a} v^{a}=0, \nabla^{a} h_{a b}^{\mathrm{TT}}=0$ and $\left(h^{\mathrm{TT}}\right)_{a}^{a}=0$. Similarly, any vector field $\zeta^{a}$ characterizing an infinitesimal gauge transformation can be decomposed as $\zeta^{a}=\nabla^{a} \zeta+V^{a}$, where $V^{a}$ is a transverse vector field. It is then clear that the vectorial and one of the scalar parts of the metric perturbation corresponding to $v^{a}$ and $\psi$ respectively can be eliminated by choosing a gauge transformation such that $V^{a}=-v^{a}$ and $\zeta=-\psi / 2$ (this will also imply a change for $\phi: \phi \rightarrow \phi+2 \square \zeta)$.

When the Lorentz gauge $\nabla^{a}\left(h_{a b}-1 / 2 \eta_{a b} h_{c}^{c}\right)=0$ is imposed, we get the following conditions on the metric perturbations: $\square v^{b}=0$ and $\nabla_{b} \phi=0$ (which implies $\phi=$ const). Any vector field characterizing the remaining gauge transformations compatible with the Lorentz gauge satisfies the condition $\square \zeta^{a}=0$, which implies $\square V^{a}=0$ and $\nabla^{a} \square \zeta=0$. We can see that a vectorial gauge transformation compatible with the Lorentz gauge can still be used to eliminate the vectorial part (now both $v^{a}$ and $V^{a}$ must be solutions of the D'Alambertian equation). On the other hand, a scalar gauge transformation such that $\square \zeta=-\phi=$ const. (this is always possible for Minkowski spacetime with a trivial-simply connected-topology) can be introduced to get $\phi=0$. Moreover, an additional scalar gauge transformation compatible with the Lorentz gauge and leaving $\phi$ invariant, which is characterized by a $\zeta$ which satisfies the D'Alambertian equation $\square \zeta=0$ [or, equivalently, $\widetilde{\zeta}(p)=0$ for $p^{2} \equiv p^{\mu} p^{\nu} \eta_{\mu \nu}$ $\neq 0$ in Fourier space], can be used to eliminate those contributions to $\psi$ which correspond to Fourier modes $\widetilde{\psi}(p)$ with $p^{2}=0$ while leaving the remaining contributions unmodified. From now on we will assume that the Lorentz gauge has been imposed and that the additional gauge transformations just mentioned have been carried out so that we are left only with the tensorial components as well as those modes of the scalar component $\psi$ with $p^{2} \neq 0$ in Fourier space.

One could select the gauge mentioned in the previous paragraph imposing suitable conditions on the reduced Wigner functional characterizing the initial state for the metric perturbations; see Appendix E for some additional comments on this point. However, as explained in Appendix D, asymptotic initial conditions should be considered in order to get a finite result for the metric correlation functions. Therefore, rather than fixing the gauge for some initial state at some finite initial time, we will work in Fourier space implicitly assuming asymptotic initial conditions and fixing the gauge as described above.

In order to analyze the two-point quantum correlation function for the metric perturbations, we will make use of the results mentioned in Sec. III and described in some more detail in Appendix C. In particular, we will exploit the fact that the stochastic correlation functions obtained with the solutions of the Einstein-Langevin equation coincide with the quantum correlation functions for the metric perturbations. Moreover, according to Eq. (14), the symmetrized twopoint quantum correlation function has two different contributions: intrinsic and induced fluctuations. We proceed now to analyze each contribution separately.

\section{Intrinsic fluctuations}

The first term on the right-hand side of Eq. (14) corresponds to the fluctuations of the metric perturbations due to the fluctuations of their initial state and is given by

$$
\left\langle\Sigma_{a b}^{(0)}(x) \Sigma_{c d}^{(0)}\left(x^{\prime}\right)\right\rangle_{\Sigma_{a b}^{(i)}, \Pi_{(i)}^{c d}},
$$


where we recall that $\Sigma_{a b}^{(0)}(x)$ is a solution of the homogeneous part of the Einstein-Langevin equation (once the Lorentz gauge has been imposed) with the appropriate initial conditions.

As mentioned in Sec. III and Appendix C, the homogeneous part of the Einstein-Langevin equation actually coincides with the linearized semiclassical Einstein equation (7). Therefore, we can make use of the results derived in Refs. $[3,16,21]$, which are briefly summarized in Appendix E. As described there, in addition to the solutions with $G_{a b}^{(1)}(x)$ $=0$, there are other solutions that in Fourier space take the form $\widetilde{G}_{\mu \nu}^{(1)}(p) \propto \delta\left(p^{2}-p_{0}^{2}\right)$ for some particular values of $p_{0}^{2}$, but they all exhibit exponential instabilities with Planckian characteristic timescales.

In order to deal with those unstable solutions, one possibility is to employ the order reduction prescription. We are then left only with the solutions which satisfy $\widetilde{G}_{\mu \nu}^{(1)}(p)=0$ (see Appendix E). The result for the metric perturbations in the gauge introduced above can be obtained by solving for the Einstein tensor in that gauge: $\widetilde{G}_{a b}^{(1)}(p)=(1 / 2) p^{2}\left[\widetilde{h}_{\mu \nu}(p)\right.$ $\left.-1 / 2 \eta_{\mu \nu} \widetilde{h}_{\rho}^{\rho}(p)\right]$. Those solutions for $\tilde{h}_{\mu \nu}(p)$ simply correspond to free linear gravitational waves propagating in Minkowski spacetime expressed in the transverse and traceless (TT) gauge. When substituting back into Eq. (16) and averaging over the initial conditions we simply get the symmetrized quantum correlation function for free gravitons in the TT gauge for the state given by the reduced Wigner function. As far as the intrinsic fluctuations are concerned, it is clear that the order reduction prescription is rather drastic, at least in the case of Minkowski spacetime, since no effects due to the interaction with the quantum matter fields are left. The method employed in Ref. [3], although slightly different, yields the same result.

A second possibility, proposed by Hawking et al. [64,65], is to impose boundary conditions which discard the runaway solutions that grow unboundedly in time and correspond to a special prescription for the integration contour when Fourier transforming back to spacetime coordinates (see Appendix E for a more detailed discussion). Following that procedure we get, for example, that for a massless conformally coupled scalar field with $\bar{\alpha}=0^{6}$ and $\bar{\beta}>0$ the intrinsic contribution to the symmetrized quantum correlation function coincides with that of free gravitons plus an extra contribution for the scalar part of the metric perturbations $\phi$ which renders Minkowski spacetime stable but plays a crucial role in providing a graceful exit for inflationary models driven by the vacuum polarization of a large number of conformal fields (such a massive scalar field would not be in conflict with present observations because, for the range of parameters usually considered, the mass would be far too large to have observational consequences [64]).

\footnotetext{
${ }^{6}$ For the massless case one can always have $\bar{\alpha}=0$ by choosing the appropriate value of the renormalization scale, as explained in Appendix B.
}

\section{Induced fluctuations}

The second term on the right-hand side of Eq. (14) corresponds to the fluctuations of the metric perturbations induced by the fluctuations of the quantum matter fields and is given by

$$
\begin{aligned}
& \frac{\bar{\kappa}^{2}}{N}\left(G_{\text {ret }} \cdot \mathcal{N} \cdot\left(G_{\text {ret }}\right)^{T}\right)_{a b c d}\left(x, x^{\prime}\right) \\
& \quad=N \kappa^{2}\left(G_{\text {ret }} \cdot \mathcal{N} \cdot\left(G_{\text {ret }}\right)^{T}\right)_{a b c d}\left(x, x^{\prime}\right),
\end{aligned}
$$

where $\mathcal{N}_{a b c d}\left(x, x^{\prime}\right)$ is the noise kernel accounting for the fluctuations of the stress tensor operator, and $\left(G_{\text {ret }}\right)_{a b c d}\left(x, x^{\prime}\right)$ is the retarded propagator with vanishing initial conditions associated with the integro-differential operator $L_{a b c d}\left(x, x^{\prime}\right)$ defined in Eq. (C10) of Appendix C.

As shown in Appendix C, the symmetrized two-point quantum correlation function coincides with the stochastic correlation function obtained from solutions of the EinsteinLangevin equation. In fact, the contribution corresponding to the induced quantum fluctuations, given by Eq. (17), is equivalent to the stochastic correlation function obtained by considering just the inhomogeneous part of the solution to the Einstein-Langevin equation: the second term on the right-hand side of Eq. (13). Taking all that into account, it is clear that we can make use of the results for the metric correlations obtained in Ref. [54] by solving the EinsteinLangevin equation (the homogeneous part of the solution was not considered there). In fact, one should simply take $N=1$ to transform our expressions to those of Ref. [54] and, similarly, multiply the noise kernel in the expressions of that reference by $N$ so that they can be used here, which follows straightforwardly from the fact that we have $N$ independent matter fields.

The same kind of exponential instabilities in the runaway solutions of the homogeneous part of the Einstein-Langevin equation (the linearized semiclassical Einstein equation) also arise when computing the retarded propagator $G_{\text {ret }}$. In order to deal with those instabilities, similar to the case of the intrinsic fluctuations, one possibility is to make use of the order reduction prescription. The Einstein-Langevin equation becomes then $G_{a b}^{(1)}=\kappa \xi_{a b}$. The second possibility, following the proposal of Hawking et al., is to impose boundary conditions which discard the exponentially growing solutions and translate into a special choice of the integration contour when Fourier transforming back to spacetime coordinates the expression for the propagator. In fact, it turns out that the propagator which results from adopting that prescription coincides with the propagator that was employed in Ref. [54]. However, it should be emphasized that this propagator is no longer the retarded one since it exhibits causality violations at Planckian scales. A more detailed discussion on all these points can be found in Appendix E.

Following Ref. [54], the Einstein-Langevin equation can be entirely written in terms of the linearized Einstein tensor $\widetilde{G}_{\mu \nu}^{(1)}(p)$ as follows:

$$
F_{\mu \nu \alpha \beta}(p) \widetilde{G}^{(1) \alpha \beta}(p)=\bar{\kappa} \widetilde{\xi}_{\mu \nu}(p),
$$


which simply corresponds to adding the stochastic source to the linearized semiclassical Einstein equation (7), where $F_{\mu \nu \alpha \beta}(p)$ was given by Eq. (8). One can then solve the stochastic equation for $\widetilde{G}_{\mu \nu}^{(1)}(p)$ and obtain its correlation function [54]:

$$
\begin{aligned}
& \left\langle\widetilde{G}_{\mu \nu}^{(1)}(p) \widetilde{G}_{\rho \sigma}^{(1)}\left(p^{\prime}\right)\right\rangle_{\xi} \\
& =\bar{\kappa}^{2} \widetilde{D}_{\mu \nu \alpha \beta}(p)\left\langle\widetilde{\xi}^{\alpha \beta}(p) \widetilde{\xi}^{\gamma \delta}\left(p^{\prime}\right)\right\rangle_{\xi} \widetilde{D}_{\rho \sigma \gamma \delta}\left(p^{\prime}\right) \\
& =\frac{\bar{\kappa}^{2}}{N} \widetilde{D}_{\mu \nu \alpha \beta}(p) \widetilde{\mathcal{N}}^{\alpha \beta \gamma \delta}(p) \widetilde{D}_{\rho \sigma \gamma \delta}(-p)(2 \pi)^{4} \delta\left(p+p^{\prime}\right),
\end{aligned}
$$

In the last equality we have taken into account translational invariance. The noise kernel $\tilde{\mathcal{N}}^{\alpha \beta \gamma \delta}(p)$ is given by Eq. (B7) in Appendix B, and $\widetilde{D}_{\mu \nu \alpha \beta}(p)$ is the propagator that results from inverting $F_{\mu \nu \alpha \beta}(p)$ (see Appendix E for a discussion on the uniqueness of this propagator) and is given by

$$
\widetilde{D}_{\mu \nu \alpha \beta}(p)=\frac{1}{F_{1}(p)} \eta_{\mu(\alpha} \eta_{\beta) \nu}-\frac{F_{2}(p)}{F_{1}(p) F_{3}(p)} p^{2} P_{\mu \nu} \eta_{\alpha \beta},
$$

with $P_{\mu \nu}=\eta_{\mu \nu}-p_{\mu} p_{\nu} / p^{2}, F_{1}(p)$ and $F_{2}(p)$ given by Eqs. (9) and (10), and $F_{3}(p)=F_{1}(p)+3 p^{2} F_{2}(p)$. On the other hand, if we make use of the order reduction prescription, we get

$$
\begin{aligned}
\left\langle\widetilde{G}_{\mu \nu}^{(1)}(p) \widetilde{G}_{\rho \sigma}^{(1)}\left(p^{\prime}\right)\right\rangle_{\xi} & =\bar{\kappa}^{2}\left\langle\widetilde{\xi}_{\mu \nu}(p) \widetilde{\xi}_{\rho \sigma}\left(p^{\prime}\right)\right\rangle_{\xi} \\
& =\frac{\bar{\kappa}^{2}}{N} \widetilde{\mathcal{N}}_{\mu \nu \rho \sigma}(p)(2 \pi)^{4} \delta\left(p+p^{\prime}\right) .
\end{aligned}
$$

Note that $G_{\mu \nu}^{(1)}(p)$ is gauge invariant when perturbing a Minkowski background because the background tensor $G_{a b}^{(0)}$ vanishes and, hence, $\mathcal{L}_{\grave{\zeta}} G_{a b}^{(0)}$ also vanishes for any vector field $\vec{\zeta}$.

Finally, using the expression for the linearized Einstein tensor in the Lorentz gauge, $\widetilde{G}_{\mu \nu}^{(1)}=(1 / 2) p^{2} \widetilde{\bar{h}}_{\mu \nu}$ with $\bar{h}_{\mu \nu}$ $=h_{\mu \nu}-(1 / 2) \eta_{\mu \nu} h_{\alpha}^{\alpha}$, we obtain the correlation function for the metric perturbations in that gauge:

$$
\begin{aligned}
\left\langle\widetilde{h}_{\mu \nu}(p) \widetilde{\bar{h}}_{\rho \sigma}\left(p^{\prime}\right)\right\rangle_{\xi}= & \frac{4 \bar{\kappa}^{2}}{N} \frac{1}{\left(p^{2}\right)^{2}} \widetilde{D}_{\mu \nu \alpha \beta}(p) \tilde{\mathcal{N}}^{\alpha \beta \gamma \delta}(p) \\
& \times \widetilde{D}_{\rho \sigma \gamma \delta}(-p)(2 \pi)^{4} \delta\left(p+p^{\prime}\right)
\end{aligned}
$$

or

$$
\left\langle\widetilde{\bar{h}}_{\mu \nu}(p) \widetilde{\bar{h}}_{\rho \sigma}\left(p^{\prime}\right)\right\rangle_{\xi}=\frac{4 \bar{\kappa}^{2}}{N} \frac{1}{\left(p^{2}\right)^{2}} \widetilde{\mathcal{N}}_{\mu \nu \rho \sigma}(p)(2 \pi)^{4} \delta\left(p+p^{\prime}\right),
$$

if the order reduction prescription is employed. It should be emphasized that, contrary to the linearized Einstein tensor $G_{a b}^{(1)}$, the metric perturbation $h_{a b}$ is not gauge invariant. This should not pose a major problem provided that the gauge has been completely fixed.

The correlation functions in spacetime coordinates can be easily derived by Fourier transforming Eq. (22) or (23). However, there is apparently an infrared divergence at $p^{2}$ $=0$, at least for the massless case. For the massive case the result is finite because the noise kernel $\tilde{\mathcal{N}}^{\alpha \beta \gamma \delta}(p)$ is proportional to $\theta\left(-p^{2}-4 m^{2}\right)$, so that $m^{2}>0$ guarantees that $p^{2}$ $=0$ lies outside the domain of integration. On the other hand, in the massless case the terms of the form $p_{\mu} p_{\nu} p_{\rho} p_{\sigma} /\left(p^{2}\right)^{2}$ appearing when substituting the noise kernel in Eqs. (22) and (23), give rise to infrared divergences when computing the Fourier transform. In fact, even if we exclude the massless case, the result would be finite, but it would become larger and larger as we chose a positive but arbitrarily small mass. In any case, such an infrared divergence seems to be just a gauge artifact. ${ }^{7}$

We can conclude that, once the instabilities giving rise to the unphysical runaway solutions have been properly dealt with, the fluctuations of the metric perturbations around the Minkowski spacetime induced by the interaction with quantum scalar fields are indeed stable (if instabilities had been present, they would have led to a divergent result when Fourier transforming back to spacetime coordinates). It should be emphasized that no ultraviolet divergences related to the coincidence limit of the noise kernel appeared in the previous analysis because we implicitly assumed asymptotic initial conditions when working in Fourier space, as explained in Appendix D. Furthermore, in contrast to the intrinsic fluctuations, even when using the order reduction prescription there is still a nontrivial contribution to the induced fluctuations due to the quantum matter fields.

\section{DISCUSSION}

In this paper we make the point that an analysis of the stability of any solution of SCG with respect to small quan-

\footnotetext{
${ }^{7}$ This is suggested by the fact that neither the correlation function of the linearized Einstein tensor nor that of the linearized Riemann tensor exhibits those divergences. The finite result for the correlation function of the Einstein tensor follows immediately from Eqs. (19) and (21), whereas for the Riemann tensor the potentially divergent contributions coming from the terms proportional to $p_{\mu} p_{\nu} p_{\rho} p_{\sigma} /\left(p^{2}\right)^{2}$ in the correlation function for the metric perturbations involve exterior products with $p_{\alpha}$ and, thus, vanish (of course the finite result for the Einstein tensor could also have been inferred from the finite result for the Riemann tensor). Alternatively, one can eliminate the terms giving rise to divergences in Eqs. (22) and (23) by performing a gauge transformation of the form $\widetilde{h}_{\mu \nu}(p) \rightarrow \widetilde{h}_{\mu \nu}(p)+p_{\mu} p_{\nu} / p^{2}$, which is generated by a vector field $\tilde{\zeta}^{\mu}(p)=p^{\mu} / p^{2}$ [consisting of just a scalar part $\widetilde{\zeta}(p)=1 / p^{2}$ ]. Such a gauge transformation does not preserve the Lorentz condition. Therefore, it seems that the infrared divergence is simply indicating a singular massless limit for the Lorentz gauge in the case under consideration.
} 
tum corrections should consider not only the evolution of the expectation value of the metric perturbations around that solution, but also their fluctuations, encoded in the quantum correlation functions. Making use of a large $N$ expansion, where $N$ is the number of matter fields, the symmetrized two-point quantum correlation function for the metric perturbations can be decomposed into two distinct parts: intrinsic fluctuations due to the fluctuations of the initial state of the metric perturbations itself and fluctuations induced by their interaction with the matter fields. The stability of the first contribution turns out to be closely related to the stability of linearized perturbations of the semiclassical Einstein equation, whereas the second contribution is equivalent to the stochastic correlation functions in stochastic semiclassical gravity obtained from solutions of the Einstein-Langevin equation.

As a specific example, we analyzed the two-point quantum correlation function for the metric perturbations around the Minkowski spacetime interacting with $N$ scalar fields initially in the Minkowski vacuum state. Once the ultraviolet instabilities (discussed in Appendix E) which are ubiquitous in SCG and are commonly regarded as unphysical have been properly dealt with by using the order reduction prescription or the procedure proposed in Refs. [64,65], both the intrinsic and the induced contributions to the quantum correlation function for the metric perturbations are found to be stable. In fact, one gets an infrared divergence for the massless case when computing the inverse Fourier transform for the induced contribution to the correlation function of the metric, but that seems to be purely a gauge effect, as argued in footnote 7 .

The symmetrized quantum correlation function for the metric perturbations obtained is in agreement with the real part of the propagator obtained by Tomboulis in Ref. [67] using a large $N$ expansion ${ }^{8}$ (he actually considered fermionic rather than scalar fields, but that just amounts to a change in one coefficient). Tomboulis used the in-out formalism rather than the CTP formalism employed in this paper. Nevertheless, his propagator is equivalent to the time-ordered CTP propagator when asymptotic initial conditions are considered because in Minkowski spacetime there is no real particle creation and the in and out vacua are equivalent (up to some phase which is absorbed in the usual normalization of the in-out propagator). The use of a CTP formulation is, however, crucial to obtaining true correlation functions rather than transition matrix elements in dynamical (nonstationary) situations (such as in an expanding Robertson-Walker background geometry), where the in-out scattering matrix might not even be well defined at all.

As we pointed out in the Introduction, Ford and collaborators have stressed the importance of the metric fluctuations and investigated some of their physical implications [4-11]. They have considered both intrinsic [6,9-11] and induced

\footnotetext{
${ }^{8}$ The imaginary part can be easily obtained from the expectation value for the commutator of the metric perturbations, which is given by $2 i \kappa\left[G_{\text {ret }}\left(x^{\prime}, x\right)-G_{\text {ret }}\left(x, x^{\prime}\right)\right]$, as briefly explained in Appendix $\mathrm{C}$.
}

fluctuations [4-8], which they usually refer to as active and passive fluctuations respectively. However, they usually consider these two kinds of fluctuations separately and have not provided a unified treatment where both of them can be understood as different contributions to the full quantum correlation function. Moreover, they always neglect the nonlocal term which encodes the averaged back reaction on the metric perturbations due to the modified dynamics of the matter fields generated by the metric perturbations themselves. ${ }^{9}$ Their justification is by arguing that those terms would be of higher order in a perturbative expansion. That is indeed the case when considering a Minkowski background if the order reduction prescription is employed, but it is not clear whether it remains true under more general conditions. In fact, as mentioned in Ref. [68], for the usual cosmological inflationary models the contribution of the nonlocal terms can be comparable or even larger than that of the remaining terms. Finally, in order to deal with the singular coincidence limit of the noise kernel, in Ref. [5] Kuo and Ford opted to subtract a number of terms including the fluctuations for the Minkowski vacuum. Even when no such subtraction was performed (because a method based on multiple integrations by parts was used instead) $[6,69,70]$, they usually discard the fluctuations for the Minkowski vacuum. Therefore, the information on the metric fluctuations around a Minkowski background when the matter fields are in the vacuum state is missing in their work.

We close this section by recalling a couple of partially open issues for which either a better understanding or a better treatment would be desirable. The first issue is the singular coincidence limit for the noise kernel. It seems clear that, when properly treating the noise kernel as a distribution, a finite result for the metric correlation function is obtained except for some divergent boundary terms at the initial time. There is a natural physical interpretation: the completely uncorrelated initial state that was considered becomes pathological when the number of modes of the environment is infinite. A simple way to overcome this problem and obtain a finite result for the correlation function is to switch on the interaction smoothly so that the modes of the environment with arbitrarily high frequencies become correlated with the system in a nonsingular way. However, in order to preserve the conservation of the source in the Einstein-Langevin equation, which guarantees the integrability of the equation through the Bianchi identity, the interaction has to be turned on adiabatically and asymptotically past initial conditions are required. Therefore, other procedures should be devised to address situations that require specifying the initial conditions at a finite initial time.

The other question which deserves further study is the procedure employed to deal with the runaway solutions discussed in Appendix E. The order reduction prescription is rather drastic as its net outcome is to discard entirely the contribution from the dissipation kernel (as far as the expectation value and the symmetrized two-point correlation func-

\footnotetext{
${ }^{9}$ In those references dealing with stochastic gravity this term is usually called the dissipation term by analogy with QBM models.
} 
tion are concerned), which encodes the averaged back reaction of the matter fields on the metric perturbations. As for the method employed by Hawking et al. in Refs. $[64,65]$, we find the fact that the choice of the physical solutions at a given instant of time depends on the far future somewhat unsatisfactory, and discarding solutions which grow unboundedly in time could get rid of other possible instabilities which are physically meaningful. Furthermore, it is not clear whether both procedures could be implemented in a general case.

To gain insight into some of the previous aspects, an interesting possibility is to consider an analogous situation in QED with the electromagnetic field regarded as an open quantum system interacting with an environment constituted by the charged quantum fields. In fact, the analogy between SCG and the equation for the expectation value of the electromagnetic field to leading order (order 1) in a large $N$ expansion for $N$ charged quantum fields has been discussed by a number of authors $[17,18,34]$. One step further was considered in Ref. [45], where the evolution of the expectation value of the electromagnetic field was considered to next to leading order in $1 / N$ (order $1 / N)$. The two-point quantum correlation functions (the CTP propagators) for the electromagnetic field to leading order in $1 / N$ (order $1 / N$ ), which play a crucial role there, are completely analogous to the quantum correlation functions for the metric perturbations considered here.

\section{ACKNOWLEDGMENTS}

It is a pleasure to thank Enrique Álvarez, Paul Anderson, Daniel Arteaga, Dieter Brill, Larry Ford, Carmen MolinaParís and Emil Mottola for interesting discussions. B.L.H. and A.R. are supported by NSF under Grant PHY03-00710. E.V. acknowledges support from the MICYT Research Project No. FPA-2001-3598.

\section{APPENDIX A: INTRINSIC AND INDUCED FLUCTUATIONS IN A SIMPLE QUANTUM BROWNIAN MOTION MODEL}

In this appendix we illustrate the importance of the fluctuations induced by the environment when considering the quantum fluctuations for an open system. As an example we will use a simple model which was analyzed in some detail in Ref. [59]: a linear quantum Brownian motion (QBM) model that consists of a harmonic oscillator, which will be referred to as the system, bilinearly coupled to a set of harmonic oscillators, which constitute the environment.

In Ref. [59] it was shown that a stochastic description based on a Langevin type equation could be used to gain information on the quantum properties of the open system. In particular, the symmetrized two-point quantum correlation function for the system turns out to be equivalent to the correlation function obtained in the context of the stochastic description:

$$
\frac{1}{2}\left\langle\left\{\hat{x}\left(t_{1}\right), \hat{x}\left(t_{2}\right)\right\}\right\rangle=\left\langle\left\langle X\left(t_{1}\right) X\left(t_{2}\right)\right\rangle_{\xi}\right\rangle_{X_{i}, p_{i}},
$$

where $\langle\cdots\rangle$ denotes the expectation value with respect to the quantum state of the system, $\hat{x}(t)$ is the position operator for the system in the Heisenberg picture, $\langle\cdots\rangle_{\xi}$ denotes the average over all possible realizations of the stochastic source $\xi(t)$ and $\langle\cdots\rangle_{X_{i}, p_{i}}$ is the average over all possible initial conditions for the solutions of the Langevin equation distributed according to the reduced Wigner function for the initial state of the system. The functions $X(t)$ appearing inside the stochastic averages are solutions of the Langevin equation $L \cdot X=\xi, \quad$ where $\quad L\left(t, t^{\prime}\right)=M\left(d^{2} / d t^{2}+\Omega_{\text {ren }}^{2}\right) \delta\left(t-t^{\prime}\right)$ $+H_{\text {ren }}\left(t, t^{\prime}\right)$, with $H_{\text {ren }}$ being the renormalized kernel appearing in the real part of the influence action and $\cdot \equiv \int_{t_{i}}^{t} d t$ throughout this appendix. Here $\xi(t)$ is a Gaussian stochastic source with vanishing expectation value and correlation function $\left\langle\xi(t) \xi\left(t^{\prime}\right)\right\rangle_{\xi}=N\left(t, t^{\prime}\right)$ where $N\left(t, t^{\prime}\right)$ is the noise kernel, being the kernel appearing in the imaginary part of the influence action (see Ref. [59] for further details). When the environment is initially in a thermal equilibrium state, the noise kernel is explicitly given by $N\left(t, t^{\prime}\right)$ $=\int_{0}^{\infty} d \omega I(\omega) \operatorname{coth} \beta \omega \cos \omega\left(t-t^{\prime}\right)$, where $I(\omega)$ is the spectral density function, which characterizes the frequency distribution of the oscillators in the environment.

The solution of the Langevin equation can be written as $X(t)=X_{0}(t)+\left(G_{\text {ret }} \cdot \xi\right)(t)$, where $X_{0}(t)$ is a solution of the homogeneous part of the Langevin equation which contains all the information about the initial conditions and $G_{\text {ret }}\left(t, t^{\prime}\right)$ is the retarded propagator with vanishing initial conditions. Substituting the previous expression for $X(t)$ into Eq. (A1) and using the properties of the stochastic source, one obtains the following result for the two-point quantum correlation function:

$$
\begin{aligned}
\frac{1}{2}\left\langle\left\{\hat{x}\left(t_{1}\right), \hat{x}\left(t_{2}\right)\right\}\right\rangle= & \left\langle X_{0}\left(t_{1}\right) X_{0}\left(t_{2}\right)\right\rangle_{X_{i}, p_{i}} \\
& +\left[G_{\text {ret }} \cdot N \cdot\left(G_{\text {ret }}\right)^{T}\right]\left(t_{1}, t_{2}\right),
\end{aligned}
$$

where the first contribution corresponds to the intrinsic fluctuations connected to the dispersion of the initial state of the system, and the term proportional to the noise kernel reflects the fluctuations induced by the system's interaction with the environment. Note the close analogy between Eq. (A2) and the expression for the symmetrized two-point quantum correlation function in the gravitational case, given by Eq. (14).

Let us specialize to the case of an ohmic environment, i.e. the case in which the spectral distribution function for the frequencies of the oscillators in the environment is of the form $I(\omega)=M \gamma \omega$, where $M$ is the mass of the system harmonic oscillator and $\gamma$ is some constant proportional to the square of the system-environment coupling constant. Then the kernel $H_{\text {ren }}$ becomes local with $H_{\text {ren }}\left(t, t^{\prime}\right)=M \gamma \delta^{\prime}(t$ $\left.-t^{\prime}\right)$ and the homogeneous solution $X_{0}(t)$ takes on the following simple form:

$$
\begin{aligned}
X_{0}(t)= & e^{-(\gamma / 2)\left(t-t_{i}\right)}\left[X_{i} \cos \widetilde{\Omega}\left(t-t_{i}\right)\right. \\
& \left.+\left(\frac{p_{i}}{M \widetilde{\Omega}}+\frac{\gamma}{2 \widetilde{\Omega}} X_{i}\right) \sin \widetilde{\Omega}\left(t-t_{i}\right)\right],
\end{aligned}
$$


where $\widetilde{\Omega}=\sqrt{\Omega_{\mathrm{ren}}^{2}-(\gamma / 2)^{2}}$ and we considered the underdamped case $\left(\Omega_{\text {ren }}>\gamma / 2\right)$. A similar result also holds for the overdamped case with the trigonometric functions replaced by the hyperbolic functions. As a result of the exponential factor, $X_{0}(t)$ and hence the intrinsic fluctuations will decay at times much larger than the relaxation time $2 \gamma^{-1} \cdot{ }^{10}$ In fact, if we take the limit $t_{i} \rightarrow-\infty$, the contribution to the twopoint correlation function from the intrinsic fluctuations completely vanishes and one is just left with the induced fluctuations. If the initial state of the environment were a thermal state, the dissipation kernel [the antisymmetric part of $\left.H_{\text {ren }}\left(t, t^{\prime}\right)\right]$ and the noise kernel are related by a fluctuation-dissipation relation which characterizes the balance between the noise induced by the environment and the dissipation effect so that the two-point correlation function remains bounded in time.

From the example employed in this appendix, it is clear that the induced fluctuations play an important role when considering correlation functions in open quantum systems. In fact, for asymptotically past initial conditions they become the entire contribution to the correlation function since the intrinsic fluctuations are completely damped by the dissipation. In a more general context, such as the gravitational case, the dissipation kernel will not damp the intrinsic fluctuations, but the induced ones will still play an important role.

\section{APPENDIX B: DISSIPATION AND NOISE KERNELS IN MINKOWSKI SPACETIME}

In this appendix we provide the expressions for the dissipation and noise kernels of a free real scalar field when a Minkowski background spacetime is considered and the state of the fields is the Minkowski vacuum. The details of their derivation can be found in Ref. [54]. All the expressions in this appendix are given in Fourier space and derived by making use of the translational invariance in terms of the inertial coordinates employed for the Minkowski background. Given any expression $\widetilde{A}(p)$, the corresponding expression in spacetime coordinates $A(x-y)$ can be simply obtained from

$$
A(x-y)=\int \frac{d^{4} p}{(2 \pi)^{4}} e^{i p x} \widetilde{A}(p) .
$$

The linear combination $-2(M+H)$ with $M$ and $H$ given by Eqs. (5),(6) is commonly referred to as the polarization tensor in an analysis based on linear response theory. We will use the term "dissipation kernel," by analogy with the usual terminology employed in the context of open quantum systems. ${ }^{11}$ In the case under consideration the local kernel

\footnotetext{
${ }^{10}$ The existence of such a decay still holds for the overdamped case provided that $\Omega_{\text {ren }} \neq 0$, otherwise there is a constant contribution that does not decay in time.

${ }^{11}$ Strictly speaking, the term dissipation kernel commonly refers to the antisymmetric part of the kernel $-H_{\text {ren }}$. Making an abuse of language, we will employ this term to refer to the whole kernel $-2 H_{\text {ren }}$ plus the local and symmetric kernel $-2 M_{\text {ren }}$.
}

$M_{\mathrm{ren}}^{\mu \nu \alpha \beta}(x-y)$ is proportional to the Einstein tensor and can be absorbed in a finite renormalization of the gravitational coupling constant. The expression in Fourier space for the nonlocal kernel $H_{\mathrm{ren}}^{\mu \nu \alpha \beta}(x-y)$ is

$$
\begin{aligned}
\widetilde{H}_{\mathrm{ren}}^{\mu \nu \alpha \beta}(p)= & \frac{1}{2}\left(P^{\mu \alpha} P^{\nu \beta}-\frac{1}{3} P^{\mu \nu} P^{\alpha \beta}\right)\left[\widetilde{H}_{A}(p)-2 \bar{\alpha}\right] \\
& +P^{\mu \nu} P^{\alpha \beta}\left[\widetilde{H}_{B}(p)-2 \bar{\beta}\right],
\end{aligned}
$$

where $\bar{\alpha}$ and $\bar{\beta}$ are constants which include the renormalized parameters $\alpha$ and $\beta$ appearing in Eq. (1), $P_{\mu \nu}$ is the projector orthogonal to $p^{\mu}$, given by $P_{\mu \nu}=\eta_{\mu \nu}-p_{\mu} p_{\nu} / p^{2}$, and

$$
\begin{aligned}
\widetilde{H}_{A}(p)= & \frac{1}{1920 \pi^{2}}\left\{( 1 + 4 \frac { m ^ { 2 } } { p ^ { 2 } } ) ^ { 2 } \left[-i \pi \operatorname{sgn} p^{0}\right.\right. \\
& \left.\left.\times \theta\left(-p^{2}-4 m^{2}\right) \sqrt{1+4 \frac{m^{2}}{p^{2}}}+\varphi\left(p^{2}\right)\right]-\frac{8}{3} \frac{m^{2}}{p^{2}}\right\},
\end{aligned}
$$

$$
\begin{aligned}
\widetilde{H}_{B}(p)= & \frac{1}{288 \pi^{2}}\left\{[ 3 ( \xi - \frac { 1 } { 6 } ) + \frac { m ^ { 2 } } { p ^ { 2 } } ] ^ { 2 } \left[-i \pi \operatorname{sgn} p^{0}\right.\right. \\
& \left.\left.\times \theta\left(-p^{2}-4 m^{2}\right) \sqrt{1+4 \frac{m^{2}}{p^{2}}}+\varphi\left(p^{2}\right)\right]-\frac{1}{6} \frac{m^{2}}{p^{2}}\right\},
\end{aligned}
$$

where $\xi$ is the parameter characterizing the coupling of the scalar field to the spacetime curvature through a term of the form $-(\xi / 2) R \phi^{2}$ in the matter Lagrangian, and $\varphi\left(p^{2}\right)$ is given by

$$
\begin{aligned}
\varphi\left(p^{2}\right)= & \int_{0}^{1} d \alpha \ln \left|1+\frac{p^{2}}{m^{2}} \alpha(1-\alpha)\right| \\
= & -2+\sqrt{1+4 \frac{m^{2}}{p^{2}}} \ln \left|\frac{\sqrt{1+4 \frac{m^{2}}{p^{2}}}+1}{\sqrt{1+4 \frac{m^{2}}{p^{2}}}-1}\right| \\
& \times \theta\left(1+4 \frac{m^{2}}{p^{2}}\right)+2 \sqrt{-1-4 \frac{m^{2}}{p^{2}}} \\
& \times \operatorname{arccotan}\left(\sqrt{-1-4 \frac{m^{2}}{p^{2}}}\right) \theta\left(-1-4 \frac{m^{2}}{p^{2}}\right) .
\end{aligned}
$$


Using the renormalized version of Eq. (4) in Fourier space, the dependence on the metric of the renormalized expectation value of the stress tensor operator can be written entirely in terms of the linearized Einstein tensor as follows:

$$
\begin{aligned}
\left\langle\overline{\widehat{T}^{\mu \nu}}[g+h ; p)\right\rangle_{\text {ren }} \\
=2 P^{\mu \nu}\left(-\frac{1}{3} \widetilde{H}_{A}(p)+\frac{2}{3} \bar{\alpha}-\widetilde{H}_{B}(p)+2 \bar{\beta}\right)\left(\widetilde{G}^{(1)}\right)_{\alpha}^{\alpha}(p) \\
\quad+\frac{2}{3} P_{\alpha}^{\alpha}\left[\widetilde{H}_{A}(p)-2 \bar{\alpha}\right]\left(\widetilde{G}^{(1)}\right)^{\mu \nu} .
\end{aligned}
$$

Following Ref. [54], we have employed a renormalization scheme in which the renormalization scale is fixed to $\mu^{2}$ $=m^{2}$. This is, of course, not possible for the massless case. Nevertheless, the expression for the massless case can still be obtained by adding a term $\ln \left(\mathrm{m}^{2} / \mu^{2}\right)$ to Eq. (B5) and subtracting $\left(1920 \pi^{2}\right)^{-1} \ln \left(\mu^{2} / m^{2}\right)$ and $(\xi-1 / 6)^{2}\left(96 \pi^{2}\right)^{-1} \ln \left(\mu^{2} / m^{2}\right)$ respectively from $2 \bar{\alpha}$ and $2 \bar{\beta}$ in Eqs. (B2) and (B6) before taking the limit $m^{2} \rightarrow 0$. The renormalized parameters will then depend on the arbitrary scale $\mu$. If desired, it is always possible to choose $\bar{\alpha}=0$ by fixing the renormalization scale $\mu$ to some appropriate value.

Finally, the expression for the noise kernel in Fourier space is given by

$$
\begin{aligned}
\tilde{\mathcal{N}}_{\mu \nu \rho \sigma}(p) & \frac{1}{2880 \pi} \theta\left(-p^{2}-4 m^{2}\right) \sqrt{1+\frac{4 m^{2}}{p^{2}}}\left\{\frac{1}{4}\left(1+\frac{4 m^{2}}{p^{2}}\right)^{2}\right. \\
& \times\left(p^{2}\right)^{2}\left(3 P_{\mu(\rho} P_{\sigma) \nu}-P_{\mu \nu} P_{\rho \sigma}\right)+10\left[3\left(\xi-\frac{1}{6}\right)\right. \\
& \left.\left.+\frac{m^{2}}{p^{2}}\right]^{2}\left(p^{2}\right)^{2} P_{\mu \nu} P_{\rho \sigma}\right\} .
\end{aligned}
$$

\section{APPENDIX C: STOCHASTIC AND QUANTUM CORRELATION FUNCTIONS}

It was initially believed that some kind of environmentinduced decoherence mechanism was required to realize the stochastic dynamics described by the Einstein-Langevin equation [51,52]. Later, in Ref. [59] it was shown that, even in the absence of decoherence, a stochastic description based on a Langevin type equation contains nontrivial information on fully quantum properties of simple linear open quantum systems. In particular, the reduced Wigner function of the system (see, for instance, Ref. [71] for the definition and properties of the Wigner function) can be expressed as a double average for the solutions of the Langevin equation with respect to both the different realizations of the stochastic source and the initial conditions, which are distributed according to the reduced Wigner function at the initial time. This expression can then be used to derive the master equation governing the time evolution of the reduced Wigner function (or, equivalently, the reduced density matrix). Fur- thermore, the stochastic correlation functions for the solutions of the Langevin equation are actually equivalent to quantum correlation functions for the system observables.

Although the previous results were obtained in Ref. [59] for linear open quantum systems, they can be extended to the case of nonlinear quantum field theories provided that some kind of Gaussian approximation for the corresponding influence functional is considered. In fact, in Ref. [58] it will be explained in detail how those results can indeed be shown to hold for the metric fluctuations around a given background spacetime by properly treating the gauge freedom and the corresponding dynamical constraints. More precisely, when considering $N$ free quantum matter fields weakly interacting with the gravitational field in the sense that the gravitational coupling constant times the number of fields remains constant in the large $N$ limit, the stochastic correlation functions can be shown to coincide with the leading order contribution to the quantum correlation functions of the metric perturbations in a large $N$ expansion.

Here we briefly sketch, in the context of a large $N$ expansion, some of the key aspects in the derivation of the result stated above. The details will appear in Ref. [58] and were partially included in Ref. [72].

We will consider metric perturbations around a globally hyperbolic background spacetime (that will be specialized to Minkowski spacetime at some point) regarded as an open quantum system interacting with the quantum matter fields, which constitute the environment. In particular we will consider $N$ minimally coupled free scalar fields, but the main result can be generalized to nonminimally coupled scalar fields or even vectorial and fermionic fields. The action for the combined system is the sum of the gravitational action $S_{\mathrm{g}}$ plus the action for the matter fields $S_{\mathrm{m}}$. The gravitational action is given by the usual Einstein-Hilbert term, the corresponding boundary term (which should be included to have a well-defined variational problem and will later be important) and the usual counterterms required to renormalize the divergences arising when functionally integrating the matter fields:

$$
\begin{aligned}
S_{\mathrm{g}}= & \frac{N}{2 \bar{\kappa}} \int_{\mathcal{M}} d^{4} x \sqrt{-\tilde{g}} R(\tilde{g})+\frac{N}{\bar{\kappa}} \int_{\mathcal{S}=\partial \mathcal{M}} d^{3} x \sqrt{\tilde{g}_{\mathcal{S}}} K_{a}^{a}(\tilde{g}) \\
& +(\text { counterterms }),
\end{aligned}
$$

where $\tilde{g}_{a b}=g_{a b}+h_{a b}$ is the perturbed metric, $g_{a b}$ is the background metric and the gravitational coupling constant $\kappa=8 \pi / m_{p}^{2}$ was rescaled to $\bar{\kappa} / N$ so that the product of the rescaled gravitational constant times the number of fields remains constant in the limit $N \rightarrow \infty$. The action for the matter fields is

$$
S_{\mathrm{m}}=-\sum_{j=1}^{N} \int_{\mathcal{M}} d^{4} x \sqrt{-\tilde{g}} \frac{1}{2}\left(\tilde{g}^{a b} \nabla_{a} \varphi_{j} \nabla_{b} \varphi_{j}+m^{2} \varphi_{j}^{2}\right)
$$

where $m$ is the mass of the scalar field. In fact, we will not take the limit $N \rightarrow \infty$, but rather use the expansion in $1 / N$ as a useful way to organize our computation and the contribu- 
tions that are included. At the very end one can always substitute back the rescaled gravitational constant in terms of the physical one.

The CTP generating functional for the metric perturbations, from which true expectation values and correlation functions can be obtained [35,37-41], is given by

$$
\begin{aligned}
Z_{\mathrm{CTP}}\left[J_{a b}, J_{c d}^{\prime}\right] & \\
= & \int \mathcal{D} h_{a b} \mathcal{D} h_{c d}^{\prime} e^{i S_{\mathrm{g}}[h]-i S_{\mathrm{g}}\left[h^{\prime}\right]} e^{i J \cdot h-i J^{\prime} \cdot h^{\prime}} \rho_{\mathrm{r}}\left[h_{a b}^{(i)}, h_{c d}^{\prime(i)}\right] \\
& \times \prod_{j=1}^{N} \int \mathcal{D} \varphi_{j} \mathcal{D} \varphi_{j}^{\prime} e^{i S_{\mathrm{m}}\left[\varphi_{j}, h\right]-i S_{\mathrm{m}}\left[\varphi_{j}^{\prime}, h^{\prime}\right]} \rho\left[\varphi_{j}^{(i)}, \varphi_{j}^{\prime(i)}\right],
\end{aligned}
$$

where we have used the notation $A \cdot B$ $\equiv \int d^{4} y \sqrt{-g(y)} A^{a b}(y) B_{a b}(y)$. The density matrices for the initial state of the fields and the metric perturbations, which are all assumed to be initially uncorrelated, are $\rho\left[\varphi_{j}^{(i)}, \varphi_{j}^{(i)}\right]$ and $\rho_{r}\left[h_{a b}^{(i)}, h_{c d}^{\prime(i)}\right]$ respectively. The gauge freedom in the path integrals for the metric perturbations should be properly treated, as briefly described below.

The first step is to integrate out the matter fields using the influence functional formalism of Feynman and Vernon for open quantum systems $[49,50]$. The influence action $S_{\mathrm{IF}}$ is defined as

$$
\begin{aligned}
e^{i S_{\mathrm{IF}}\left[h, h^{\prime}\right]}= & \prod_{j=1}^{N} \int \mathcal{D} \varphi_{j} \mathcal{D} \varphi_{j}^{\prime} e^{i S_{\mathrm{m}}\left[\varphi_{j}, h\right]-i S_{\mathrm{m}}\left[\varphi_{j}^{\prime}, h^{\prime}\right]} \\
& \times \rho\left[\varphi_{j}^{(i)}, \varphi_{j}^{\prime(i)}\right] .
\end{aligned}
$$

Up to quadratic order in the metric perturbations it is given by $[51,52]$

$$
S_{\mathrm{IF}}\left[\Sigma_{a b}, \Delta_{a b}\right]=N\left(Z \cdot \Delta+\Delta \cdot(H+M) \cdot \Sigma+\frac{i}{8} \Delta \cdot \mathcal{N} \cdot \Delta\right),
$$

where we have introduced the semisum and difference variables $\Sigma_{a b}=\left(h_{a b}+h_{a b}^{\prime}\right) / 2 \quad$ and $\quad \Delta_{a b}=h_{a b}^{\prime}-h_{a b}, \quad Z^{a b}(x)$ $=-(1 / 2)\left\langle\hat{T}^{a b}[\hat{\varphi}, g ; x)\right\rangle$ and the kernels $H, M$ and $\mathcal{N}$ were defined in Eqs. (5), (6) and (12). As explained in Sec. II, the kernels $H$ and $M$ exhibit divergences that are canceled by renormalizing the gravitational coupling constant and the cosmological constant in the bare gravitational action as well as the coupling constants of the counterterms quadratic in the curvature. We will not need terms of higher order in the metric perturbations because they give contributions to the connected part of the CTP generating functional (given by $\left.W_{\text {CTP }}^{(\mathrm{LO})}=-i \ln Z_{\mathrm{CTP}}^{(\mathrm{LO})}\right)$ of higher order in $1 / N$. This is also true for the terms in the gravitational action $S_{\mathrm{g}}$, which implies that we do not have to consider graviton vertices. In order to show that, when computing the connected part of the CTP generating functional to leading order in $1 / N$, it is indeed sufficient to keep just those terms in the gravitational action and the influence action which are at most quadratic in the metric perturbations, one can first compute the generating functional resulting from that approximation, $Z_{\mathrm{CTP}}^{(\mathrm{LO})}$, and then show that including terms of higher order in the metric perturbations would yield corrections to the connected part of the generating functional of higher order in $1 / N$.

We now specialize to the case of a Minkowski background, i.e. $g_{a b}=\eta_{a b}$, and consider a family of Cauchy hypersurfaces which foliate the spacetime into constant time hypersurfaces of a given inertial frame in Minkowski spacetime. The initial states for the metric perturbations and the matter fields are specified on one of these hypersurfaces, which will be denoted by $\mathcal{S}_{i}$. Another hypersurface is chosen as the final hypersurface $\mathcal{S}_{f}$ so that any spacetime region of interest lies between them. Then we integrate by parts those contributions to the Einstein-Hilbert term of the gravitational action involving two derivatives acting on the same factor and impose the Lorentz gauge condition $\nabla^{a} \bar{h}_{a b}=0$ [we recall that the indices are raised and lowered using the background Minkowski metric, all the covariant derivatives are taken in this background metric and $\left.\bar{A}_{a b} \equiv A_{a b}-(1 / 2) \eta_{a b} A_{c}^{c}\right]$. The boundary terms resulting from integration by parts are canceled by the boundary terms included in the gravitational action $^{12}$ and the expression $\widetilde{S}_{\mathrm{g}}\left[h, h^{\prime}\right]=S_{\mathrm{g}}[h]-S_{\mathrm{g}}\left[h^{\prime}\right]$ up to quadratic order in the metric perturbations becomes

$$
\begin{aligned}
\widetilde{S}_{\mathrm{g}}\left[\Sigma_{a b}, \Delta_{c d}\right]= & \frac{N}{4 \bar{\kappa}} \int_{\mathcal{M}} d^{4} x \sqrt{-g} \nabla_{a} \Delta^{b c} \nabla^{a} \bar{\Sigma}_{b c} \\
& +(\text { counterterms }) .
\end{aligned}
$$

Next, we introduce the momentum canonically conjugate to $\Delta_{a b},{ }^{13}$ which is given by

$$
\Pi^{a b}\left[\Sigma_{c d}\right]=\frac{\delta \widetilde{S}_{\mathrm{g}}}{\delta \dot{\Delta}_{a b}}=-\frac{N}{4 \bar{\kappa}} \dot{\bar{\Sigma}}^{a b},
$$

where we employed the notation $\dot{A}_{a b} \equiv n^{c} \nabla_{c} A_{a b}$ for the covariant derivative with respect to the normalized and futuredirected timelike vector $n^{a}$ orthogonal to the family of Cauchy hypersurfaces including the initial and final hypersurfaces $\mathcal{S}_{i}$ and $\mathcal{S}_{f}$. Finally, one can integrate again by parts so that

\footnotetext{
${ }^{12}$ In general, one should be careful with the contributions from the timelike boundaries as well as the edges connecting the spacelike and timelike boundaries [79]. Here we will assume that the timelike boundaries are infinitely far away and the value of the metric perturbations decays at large distances so that only the contributions from the spacelike boundaries are relevant.

${ }^{13}$ Throughout this section we will neglect the contribution to the momentum from the counterterms; see Appendix E for further discussion of this point.
} 


$$
\begin{aligned}
S_{\mathrm{g}}\left[\Sigma_{a b}, \Delta_{c d}\right]= & -\frac{N}{4 \bar{\kappa}} \int_{\mathcal{M}} d^{4} x \sqrt{-g} \Delta^{b c} \nabla_{a} \nabla^{a} \bar{\Sigma}_{b c} \\
& +\int_{\mathcal{S}_{f} \subset \partial \mathcal{M}} d^{3} x \sqrt{g_{\mathcal{S}_{f}}} \Pi_{(f)}^{a b} \Delta_{a b}^{(f)} \\
& -\int_{\mathcal{S}_{i} \subset \partial \mathcal{M}} d^{3} x \sqrt{g_{\mathcal{S}_{i}}} \Pi_{(i)}^{a b} \Delta_{a b}^{(i)}+(\text { counterterms }),
\end{aligned}
$$

where the indices $(i)$ and $(f)$ denote quantities evaluated on $\mathcal{S}_{i}$ and $\mathcal{S}_{f}$ respectively. It should be emphasized that $h_{a b}^{(i)}$ or $h_{a b}^{(f)}$ simply correspond to the spacetime metric evaluated on those hypersurfaces and should not be confused with the induced metric in the usual Arnowitt-Deser-Misner (ADM) formulation. Furthermore, the contribution for $\mathcal{S}_{f}$ will not be relevant because, when computing the CTP generating functional, we should take $h_{a b}=h_{a b}^{\prime}$ (which implies $\left.\Delta_{a b}=0\right)$ on the final hypersurface.

Changing to the new current variables $J_{a b}^{\Sigma}=\left(J_{a b}\right.$ $\left.+J_{a b}^{\prime}\right) / 2$ and $J_{a b}^{\Delta}=J_{a b}^{\prime}-J_{a b}$, and functionally integrating with respect to $\Delta_{c d}$, one gets the following expression for the generating functional:

$$
\begin{aligned}
Z_{\mathrm{CTP}}^{(\mathrm{LO})}\left[J_{a b}^{\Sigma}, J_{c d}^{\Delta}\right] & =K \int \mathcal{D} \Sigma_{a b} \exp \left[-\frac{N}{2 \bar{\kappa}^{2}}\left(L \cdot \Sigma-\frac{2 \bar{\kappa}}{N} J^{\Sigma}\right) \cdot \mathcal{N}^{-1} \cdot(L \cdot \Sigma\right. \\
& \left.\left.-\frac{2 \bar{\kappa}}{N} J^{\Sigma}\right)\right] e^{i J^{\Delta} \cdot \Sigma} W_{\mathrm{r}}\left[\Sigma_{a b}^{(i)}, \Pi_{(i)}^{c d}\right],
\end{aligned}
$$

where the functional integral with respect to $\Sigma_{a b}$ is restricted to those configurations that satisfy the Lorentz gauge condition, and we introduced the integro-differential operator

$$
\begin{aligned}
L_{a b c d}\left(x, x^{\prime}\right)= & (1 / 2)\left(\eta_{a c} \eta_{b d}-\eta_{a b} \eta_{c d} / 2\right) \square \delta\left(x-x^{\prime}\right) \\
& +2 \bar{\kappa} H_{a b c d}^{(\mathrm{ren})}\left(x-x^{\prime}\right)+2 \bar{\kappa} M_{a b c d}^{(\mathrm{ren})}\left(x-x^{\prime}\right) .
\end{aligned}
$$

$K$ is some normalization constant which can be eventually determined by demanding that $Z_{\mathrm{CTP}}^{(\mathrm{LO})}\left[J_{a b}^{\Sigma}, J_{c d}^{\Delta}\right]=1$ when we take $J_{a b}^{\Sigma}=J_{c d}^{\Delta}=0$, and $W_{\mathrm{r}}\left[\Sigma_{a b}^{(i)}, \Pi_{(i)}^{c d}\right]$ is the reduced Wigner functional for the metric perturbations at the initial time, which is defined in terms of the reduced density matrix at the initial time as

$W_{\mathrm{r}}\left[\Sigma_{a b}^{(i)}, \Pi_{(i)}^{c d}\right]=(1 / 2 \pi) \int d \Delta_{c d}^{(i)} \exp \left(i \Pi_{(i)}^{c d} \Delta_{c d}^{(i)}\right) \rho_{\mathrm{r}}\left[\Sigma_{a b}^{(i)}, \Delta_{c d}^{(i)}\right]$

The Lorentz gauge condition does not fix completely the gauge freedom under local diffeomorphisms. However, it can be completely fixed by imposing additional gauge fixing conditions on the state of the metric perturbations at the initial time. Thus, from now on it should be understood that some appropriate condition such as the transverse and trace- less gauge has been imposed on the reduced density matrix (or, equivalently, the reduced Wigner functional) at the initial time.

Introducing a suitable functional change, the CTP generating functional can be rewritten in the following way when taking $J_{a b}^{\Sigma}=0$ :

$$
Z_{\mathrm{CTP}}^{(\mathrm{LO})}\left[J_{a b}^{\Sigma}=0, J_{c d}^{\Delta}\right]=\left\langle\left\langle e^{i J^{\Delta} \cdot \Sigma}\right\rangle_{\xi}\right\rangle_{\Sigma_{a b}^{(i)}, \Pi_{(i)}^{c d}},
$$

where the expectation values $\langle\cdots\rangle_{\Sigma_{a b}^{(i)}, \Pi_{(i)}^{c d}}$ and $\langle\cdots\rangle_{\xi}$ are defined as

$$
\begin{aligned}
\langle\cdots\rangle_{\Sigma_{a b}^{(i)}, \Pi_{(i)}^{c d}} & =\int d \Sigma_{a b}^{(i)} d \Pi_{(i)}^{c d} \cdots W_{\mathrm{r}}\left[\Sigma_{a b}^{(i)}, \Pi_{(i)}^{c d}\right], \\
\langle\cdots\rangle_{\xi} & =(2 \pi \mathcal{N} / N)^{-1 / 2} \int \mathcal{D} \xi_{a b} \cdots e^{-(N / 2) \xi \cdot \mathcal{N}^{-1} \cdot \xi} .
\end{aligned}
$$

The $\Sigma_{a b}(x)$ inside the expectation values in Eq. (C12) satisfies the equation

$$
(L \cdot \Sigma)_{a b}(x)=\bar{\kappa} \xi_{a b}(x),
$$

with initial conditions $\Sigma_{a b}^{(i)}$ and $\Sigma_{a b}^{(i)}=-(4 \bar{\kappa} / N) \bar{\Pi}_{(i)}^{c d}$ on the initial hypersurface $\mathcal{S}_{i}$. From Eq. (C14) it becomes clear that one can formally interpret $\xi_{a b}$ as a Gaussian stochastic source with a vanishing expectation value and whose correlation function is given by the noise kernel. Equation (C15) can then be regarded as a stochastic Langevin equation and coincides with the Einstein-Langevin equation expressed in the Lorentz gauge (when integrated with the metric perturbation $h_{a b}$, the first term on the right-hand side of Eq. (C10) corresponds to the linearized Einstein tensor, whereas the last two terms correspond to $\left\langle\hat{T}_{a b}^{(1)}[g+h]\right\rangle$, as follows from Eq. (4)). Furthermore, $Z_{\mathrm{CTP}}^{(\mathrm{LO})}\left[J_{a b}^{\Sigma}=0, J_{c d}^{\Delta}\right]$ is also the generating functional for the stochastic correlation functions for the solutions of the Einstein-Langevin equation and, therefore, the stochastic correlation functions are actually equivalent to quantum correlation functions for the metric perturbations.

The solutions of Eq. (C15) can be expressed as

$$
\Sigma_{a b}(x)=\Sigma_{a b}^{(0)}(x)+\bar{\kappa}\left(G_{\mathrm{ret}} \cdot \xi\right)_{a b}(x)
$$

where $\Sigma_{a b}^{(0)}$ is a solution of the homogeneous equation $(L \cdot \Sigma)_{a b}=0$, which coincides with the linearized semiclassical Einstein equation for the metric perturbations in the Lorentz gauge, with all the information on the initial conditions, and $\left(G_{\text {ret }}\right)_{a b c d}\left(x, x^{\prime}\right)$ is the retarded propagator associated with the integro-differential operator $L_{a b c d}\left(x, x^{\prime}\right)$ with vanishing initial conditions on $\mathcal{S}_{i}$. The CTP generating functional for a nonvanishing $J_{a b}^{\Sigma}$ can then be written as follows: 


$$
\begin{aligned}
Z_{\mathrm{CTP}}^{(\mathrm{LO})}\left[J_{a b}^{\Sigma}, J_{c d}^{\Delta}\right] \\
=\left\langle e^{i J^{\Delta} \cdot \Sigma^{(0)}}\right\rangle_{\Sigma_{a b}^{(i)}, \Pi_{(i)}^{c d}} \\
\quad \times \exp \left(i \frac{2 \bar{\kappa}}{N} J^{\Delta} \cdot G_{\mathrm{ret}} \cdot J^{\Sigma}\right) \\
\quad \times \exp \left(-\frac{\bar{\kappa}^{2}}{2 N} J^{\Delta} \cdot G_{\mathrm{ret}} \cdot \mathcal{N} \cdot G_{\mathrm{ret}}^{T} \cdot J^{\Delta}\right),
\end{aligned}
$$

where we introduced the notation $\left(A^{T}\right)_{a b c d}(x, y)$ $\equiv A_{c d a b}(y, x)$.

It is interesting to consider the particular case of the twopoint correlation function. Functionally differentiating twice with respect to $J_{a b}^{\Delta}$ and then taking $J_{a b}^{\Delta}$ and $J_{a b}^{\Sigma}$ equal to zero, one gets the following result for the symmetrized quantum correlation function for the metric perturbations:

$$
\begin{aligned}
\frac{1}{2}\left\langle\left\{\hat{h}_{a b}(x), \hat{h}_{c d}\left(x^{\prime}\right)\right\}\right\rangle= & \left\langle\Sigma_{a b}^{(0)}(x) \Sigma_{c d}^{(0)}\left(x^{\prime}\right)\right\rangle_{\Sigma_{a b}^{(i)}, \Pi_{(i)}^{c d}} \\
& +\frac{\bar{\kappa}^{2}}{N}\left(G_{\mathrm{ret}} \mathcal{N} \cdot\left(G_{\mathrm{ret}}\right)^{T}\right)_{a b c d}\left(x, x^{\prime}\right) .
\end{aligned}
$$

One can see that there are two separate contributions to the two-point correlation function: the first one is related to the dispersion of the initial state for the metric perturbations, whereas the second one is proportional to the noise kernel and accounts for the fluctuations induced by their interaction with the environment (in this case, the quantum matter fields). We refer to these two contributions as intrinsic and induced fluctuations respectively. Furthermore, taking into account Eq. (C12), we see that, under the aforementioned conditions, the symmetrized quantum correlation function for the metric perturbations is equivalent to the stochastic correlation function obtained in stochastic semiclassical gravity by solving the Einstein-Langevin equation.

From the expression for the generating functional in Eq. (C17) one can get the remaining two-point quantum correlation functions to leading order in $1 / N$. In particular the commutator is given by $\left\langle\left[\hat{h}_{a b}(x), \hat{h}_{c d}\left(x^{\prime}\right)\right]\right\rangle=2 i \kappa\left(G_{\mathrm{ret}}\left(x^{\prime}, x\right)\right.$ $\left.-G_{\text {ret }}\left(x, x^{\prime}\right)\right)$, and by combining the commutator and the anticommutator the rest of two-point functions can be easily obtained. Moreover, assuming a Gaussian initial state with vanishing expectation value for the metric perturbations, the expression for the generating functional in Eq. (C17) becomes Gaussian and any other $n$-point quantum correlation function has a simple expression in terms of the two-point functions.

The exact CTP generating functional is given by

$$
Z_{\mathrm{CTP}}\left[J_{a b}^{\Sigma}, J_{c d}^{\Delta}\right]=\exp \left(i S_{\mathrm{int}}\left[\frac{\delta}{\delta J_{a b}^{\Sigma}}, \frac{\delta}{\delta J_{c d}^{\Delta}}\right]\right) Z_{\mathrm{CTP}}^{(\mathrm{LO})}\left[J_{a b}^{\Sigma}, J_{c d}^{\Delta}\right],
$$

where $S_{\text {int }}\left[\Sigma_{a b}, \Delta_{c d}\right]$ corresponds to all the terms in the gravitational action or the exact influence action of cubic or
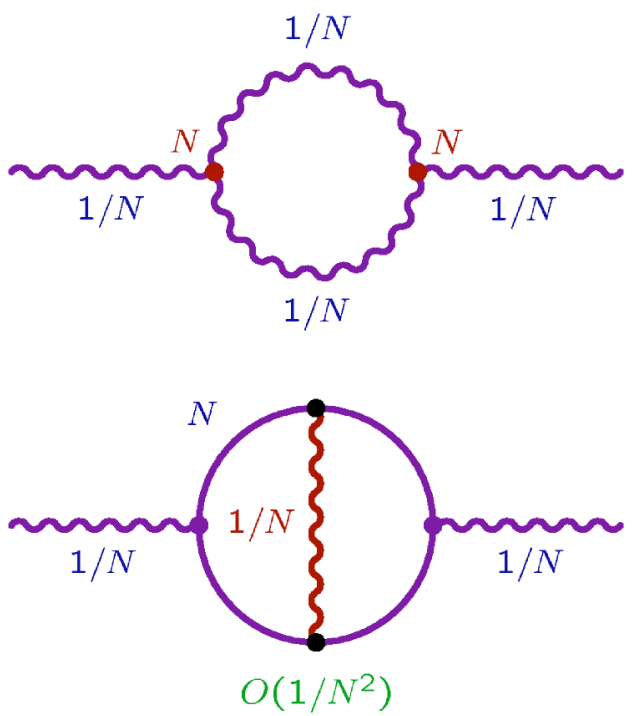

FIG. 1. Two diagrams illustrating the fact that including either the vertices for the metric perturbations (as in the first diagram) or terms from the influence functional evaluated beyond the Gaussian approximation (as in the second diagram) lead to contributions of higher order in $1 / N$. In particular, the two diagrams shown here give contributions of order $1 / N^{2}$ to the two-point quantum correlation function for the metric perturbations. The plain lines represent the CTP propagators for the matter fields on the background spacetime and the wavy lines correspond to the CTP propagators for the metric perturbations obtained by functionally differentiating $W_{\mathrm{CTP}}^{(\mathrm{LO})}$ twice with respect to the external currents.

higher order in the metric perturbations. In order to consider and evaluate the different contributions to Eq. (C19), it is convenient to introduce the corresponding Feynman rules and diagrams (in the CTP formulation) as follows: each term in $S_{\text {int }}\left[\delta / \delta J_{a b}^{\Sigma}, \delta / \delta J_{c d}^{\Delta}\right]$ gives rise to a vertex with the same number of legs as the total power of the functional derivatives $\delta / \delta J_{a b}^{\Sigma}$ and $\delta / \delta J_{c d}^{\Delta}$ appearing in that term, and the CTP propagators simply correspond to those obtained by functionally differentiating $W_{\mathrm{CTP}}^{(\mathrm{LO})}=-i \ln Z_{\mathrm{CTP}}^{\mathrm{LO})}$ with respect to the external currents twice. Expanding in powers of $1 / N$, one can show that all the diagrams representing the corrections, as given by Eq. (C19), to the connected part of the generating functional, $W_{\mathrm{CTP}}=-i \ln Z_{\mathrm{CTP}}$, are of order $1 / N^{2}$ or higher [58]. Therefore, one can conclude that the leading order contribution to $W_{\mathrm{CTP}}$ is entirely given by $W_{\mathrm{CTP}}^{(\mathrm{LO})}$, which is of order $1 / N$ and from which the leading order contribution to all the quantum correlation functions with an even number of points can be obtained. Two particular examples showing how the corrections due to $S_{\text {int }}\left[\Sigma_{a b}, \Delta_{c d}\right]$ contribute to the two-point quantum correlation functions (corresponding to terms in $W_{\text {CTP }}$ which are quadratic in the external currents) are provided in Fig. 1. The first diagram involves vertices with three legs associated with cubic terms in the gravitational action. The second diagram involves a nonlocal vertex with four legs associated with quartic terms in the influence action. The nonlocal vertex has been represented by a loop of the matter fields because, if Feynman diagrams are introduced when evaluating the influence action, the terms quartic in the metric perturbations giving rise to the second diagram in Fig. 1 correspond to a loop of matter 
fields with four insertions linear in the metric perturbations (there are three other contributions to the influence action involving terms quartic in the metric perturbations: one corresponds to a loop of matter fields with two insertions linear in the metric perturbation and a third insertion quadratic in the metric perturbation, a second contribution that corresponds to a loop of matter fields with two insertions quadratic in the metric perturbation, and a third one corresponding to a loop of matter fields with an insertion linear in the metric perturbation and a second insertion cubic in the metric perturbation).

\section{APPENDIX D: SINGULAR COINCIDENCE LIMIT FOR THE NOISE KERNEL}

The noise kernel defined by Eq. (12) has in general a singular coincidence limit $x \rightarrow x^{\prime}$ (in fact, it is still singular even for $x \neq x^{\prime}$ when the two points are connected by a null geodesic), which translates into an ultraviolet divergence when integrating over momenta in Fourier space, as can be seen from Eq. (B7). The result is, nevertheless, finite when $x \neq x^{\prime}$ (and they are not connected by a null geodesic). In fact, even though the noise kernel is not well defined as a tensor-valued function, ${ }^{14}$ it is well defined as a tensor-valued distribution and yields finite results when integrated with suitable test functions.

Let us consider a specific example to illustrate the points addressed in this appendix: a massless conformally coupled scalar field in Minkowski spacetime. The expression for the noise kernel in spacetime coordinates, which results from Fourier transforming Eq. (B7) and is a well-defined distribution, is the following [54]:

$$
\begin{aligned}
\mathcal{N}_{\mu \nu \rho \sigma}\left(x-x^{\prime}\right) & \propto\left(\partial_{\mu} \partial_{\nu} \partial_{\rho}^{\prime} \partial_{\sigma}^{\prime}\right) \mathcal{P} f\left(\frac{1}{\left(x-x^{\prime}\right)^{2}}\right)^{2} \\
= & \frac{1}{16}\left(\partial_{\mu} \partial_{\nu} \partial_{\rho}^{\prime} \partial_{\sigma}^{\prime}\right) \square_{x} \square_{x}^{\prime} \ln \left(x-x^{\prime}\right)^{2},
\end{aligned}
$$

where $\mathcal{P} f$ stands for the Hadamard finite part prescription, whose precise definition can be found in Refs. [73,74]. However, the contribution from the induced fluctuations to the symmetrized two-point correlation function, which is given by

$$
\frac{\bar{\kappa}^{2}}{N}\left[G_{\text {ret }} \cdot \mathcal{N} \cdot\left(G_{\text {ret }}\right)^{T}\right]\left(x_{1}, x_{2}\right),
$$

is not necessarily well defined if the time integral in the center dot $(\cdot)$ involves a finite initial time $t_{i}$. That is because in that case the noise kernel is actually convoluted with $G_{\text {ret }}\left(x_{1}, x_{1}^{\prime}\right) \theta\left(t_{1}^{\prime}-t_{i}\right)$, which is not a good test function since it is not differentiable at $t_{1}^{\prime}=t_{i}$. The fact that the result for

\footnotetext{
${ }^{14}$ The noise kernel is in general a bitensor, but due to the homogeneity of Minkowski spacetime and the triviality of the connection (and the corresponding parallel transport), the noise kernel becomes in that case a simple tensorial field which depends on $\left(x-x^{\prime}\right)^{\mu}$.
}

expression (D2) is singular for a finite initial time can actually be seen by using the last equality in Eq. (D1), substituting into expression (D2) and integrating by parts. The contributions from the boundary terms at the finite times $t_{1}$ and $t_{2}$ are finite, at least when $t_{1} \neq t_{2}$ [the fact that $t_{1} \neq t_{2}$ may be required to get a finite result is simply indicating that expression (D2) is also a distribution]. On the other hand, the boundary terms that correspond to taking $t_{1}^{\prime}$ and $t_{2}^{\prime}$ both equal to $t_{i}$ are divergent. The fact that all the singular contributions can be concentrated at the initial time seems to suggest that the origin of the problem may be related to the initial state that was chosen.

We proceed now to argue that the origin of the singularities described in the previous paragraph can indeed be traced back to the initial state that was considered, with the metric perturbations and the matter fields completely uncorrelated. In order to do that, it will be useful to discuss an analogous situation for QBM models such as that described in Appendix A. In particular, let us consider an Ohmic distribution for the environment frequencies with an ultraviolet cutoff $\Lambda$, which can be characterized by a spectral density function such as $I(\omega)=\omega \theta(\Lambda-\omega)$ or $\omega \exp (-\omega / \Lambda)$ (the details about the particular way in which the cutoff is implemented are not important here). We have an expression analogous to Eq. (D2) for the induced fluctuations [see Eq. (A2) and Ref. [59]]. If we consider the ground state (thermal state at zero temperature) as the initial state for the environment, the noise kernel is given by $N\left(t, t^{\prime}\right)=\int d \omega I(\omega) / \omega \cos \omega\left(t-t^{\prime}\right)$. When taking the limit $\Lambda \rightarrow \infty$ the noise kernel becomes proportional to $\mathcal{P} f\left(1 /\left(t_{1}^{\prime}-t_{2}^{\prime}\right)^{2}\right)$. To obtain the correlation function we integrate by parts, as described above, in the expression for the induced fluctuations, Eq. (A2), before taking the limit $\Lambda \rightarrow \infty$. We obtain again a boundary term at the initial time which diverges as we finally let $\Lambda$ go to infinity, and we end up with an infinite result for the correlation function. On the other hand, one can show that the result for the correlation function of the ground state of the whole system (system plus environment), including the system-environment interaction, is finite (the use of Euclidean path integrals is particularly convenient in this respect) [75]. This constitutes a clear example of the fact that initial states in which the system and the environment are suitably correlated give rise to well-defined correlation functions.

Alternatively, when taking a completely uncorrelated initial state, one can still get a finite result for the correlation function by smoothly switching on the system-environment interaction so that the boundary term at the initial time which results from the integration by parts and becomes divergent in the limit $\Lambda \rightarrow \infty$ actually vanishes. This reveals again that the origin of the singularity for the correlation function arises because the highest frequency modes of the environment become correlated with the system in a time scale of the order of $\Lambda^{-1}$. Such a fact is supported by the existence of a jolt with a characteristic time scale $\Lambda^{-1}$ in the diffusion coefficients of the master equation which becomes singular when $\Lambda \rightarrow \infty$, as was found in Ref. [76]. In fact, one can show that those states in which the high frequency modes of the environment and the system are uncorrelated are unphysical when the environment contains an infinite number of modes 
with arbitrarily high frequencies since their energy becomes infinite as $\Lambda \rightarrow \infty$.

Return now to the gravitational case. There are some situations, such as the effect of stress tensor fluctuations on the propagation of null geodesics, in which the appropriate way to deal with the singular coincidence limit of the noise kernel is by integrating over some smearing function [8] (in general smearing just along the spatial directions is not enough: smearing in time is needed to get a finite result). On the other hand, when computing the correlation functions for the metric perturbations, the noise kernel naturally appears integrated with the retarded propagator. As explained above, the problem still persists at the initial time, which reflects the unphysical character of the completely uncorrelated initial state that was employed. Similar to QBM models, a welldefined result for the correlation functions can be obtained by considering a properly correlated initial state, such as that resulting from the use of Euclidean path integrals that are then analytically continued to Lorentzian time [64]. Roughly speaking, this would imply the existence of an additional term in Eqs. (14) and (C18) due to the existence of correlations between the initial conditions for the solutions of the Langevin equation and the stochastic source, which reflect the initial correlations between the system and the environment.

Alternatively, one can still make sense of the results obtained from assuming an uncorrelated initial state by smoothly switching on the interaction between the metric perturbations and the matter fields so that the high frequency modes can get correlated with the system. However, in contrast to the QBM case, we have to be careful with switching on the interaction during a finite period of time since that would imply that the source of the Einstein-Langevin equation is not conserved and would be in conflict with the Bianchi identity, which guarantees the integrability of the equation. Therefore, the interaction should be turned on adiabatically and asymptotically past initial conditions should be considered. In fact, in Sec. IV, where we assumed asymptotic initial conditions and worked mostly in Fourier space, a finite result for the correlation function was obtained without the need for explicitly switching on the interaction adiabatically. There are, however, situations (for instance, in cosmology) in which asymptotic initial conditions are not adequate. An alternative procedure should be considered in those cases.

\section{APPENDIX E: RUNAWAY SOLUTIONS AND METHODS TO DEAL WITH THEM}

In this appendix we will briefly discuss the existence of runaway solutions in SCG (solutions which grow without bound in time scales comparable to the Planck time), their counterparts at the quantum level, and how their connection can be understood in the context of stochastic gravity. We will also discuss the existing prescriptions for dealing with this kind of unstable solution.

\section{Runaway solutions in semiclassical gravity}

Let us start by considering the linearized semiclassical Einstein equation around the Minkowski spacetime. The so- lutions for the case of a massless scalar field were first discussed in Ref. [16] and an exhaustive description can be found in Appendix A of Ref. [21]. Taking Eq. (7) and using a decomposition for the linearized Einstein tensor analogous to that introduced in Sec. IV C for the metric perturbation, the vectorial part is found to vanish, ${ }^{15}$ whereas the scalar and tensorial contributions satisfy the equations

$$
\begin{aligned}
{\left[F_{1}(p)+3 p^{2} F_{2}(p)\right] \widetilde{G}_{\mu \nu}^{(1)(\mathrm{S})}(p) } & =0, \\
F_{1}(p) \widetilde{G}_{\mu \nu}^{(1)(\mathrm{T})}(p) & =0 .
\end{aligned}
$$

where $F_{1}(p)$ and $F_{2}(p)$ are given by Eqs. (9) and (10), and $\widetilde{G}_{\mu \nu}^{(1)(\mathrm{S})}$ and $\widetilde{G}_{\mu \nu}^{(1)(\mathrm{T})}$ denote, respectively, the scalar and tensorial parts of the linearized Einstein tensor. In order to illustrate how the runaway solutions arise, we will consider the particular example of a massless and conformally coupled scalar field (see Ref. [21] for the massless case with arbitrary coupling and Refs. $[3,54]$ for the general massive case). The previous equations become then

$$
\begin{gathered}
\left(1+12 \kappa \bar{\beta} p^{2}\right) \widetilde{G}_{\mu \nu}^{(1)(\mathrm{S})}(p)=0, \\
\lim _{\epsilon \rightarrow 0^{+}}\left[1+\left(960 \pi^{2}\right)^{-1} \kappa p^{2} \ln \left(\frac{-\left(p^{0}+i \epsilon\right)^{2}+\vec{p}^{2}}{\mu^{2}}\right)\right] \widetilde{G}_{\mu \nu}^{(1)(\mathrm{T})}(p) \\
=0 .
\end{gathered}
$$

In addition to the obvious solution $\widetilde{G}_{\mu \nu}^{(1)(\mathrm{S})}(p)=0$ (the only solution when $\bar{\beta}=0$ ), when $\bar{\beta}>0$ the solutions for the scalar component exhibit an oscillatory behavior in spacetime coordinates which corresponds to a massive scalar field with $m^{2}=(12 \kappa|\bar{\beta}|)^{-1}$; for $\bar{\beta}<0$ the solutions correspond to a tachyonic field with $m^{2}=-(12 \kappa|\bar{\beta}|)^{-1}$ : in spacetime coordinates they exhibit an exponential behavior in timegrowing or decreasing-for wavelengths larger than $4 \pi(3 \kappa|\bar{\beta}|)^{1 / 2}$ and an oscillatory behavior for wavelengths smaller than $4 \pi(3 \kappa|\bar{\beta}|)^{1 / 2}$. On the other hand, the solution $\widetilde{G}_{\mu \nu}^{(1)(S)}(p)=0$ is completely trivial since any scalar metric perturbation $\widetilde{h}_{\mu \nu}(p)$ giving rise to a vanishing linearized Einstein tensor can be eliminated by a gauge transformation as explained in Sec. IV C.

As for the tensorial component, when $\mu \leqslant \mu_{\text {crit }}$ $=l_{p}^{-1}(120 \pi)^{1 / 2} e^{\gamma}$ (or $\lambda \geqslant \lambda_{\text {crit }}=\mu_{\text {crit }}^{-1}$ in the notation of Ref. [21]) the first factor in Eq. (E4) vanishes for four complex values of $p^{0}$ of the form $\pm \omega$ and $\pm \omega^{*}$, where $\omega$ is some complex value, as illustrated in Fig. 2. We will consider here the case in which $\mu<\mu_{\text {crit }}$; a detailed description of the situation for $\mu \geqslant \mu_{\text {crit }}$ can be found in Appendix A of Ref.

\footnotetext{
${ }^{15}$ More precisely, decomposing the metric perturbation into scalar, vectorial and tensorial parts, as done in Sec. IV C, and computing the linearized Einstein tensor, one gets a vanishing result for the vectorial part of the metric perturbation; the scalar and tensorial components of the metric perturbation give rise, respectively, to the scalar and tensorial components of the linearized Einstein tensor.
} 


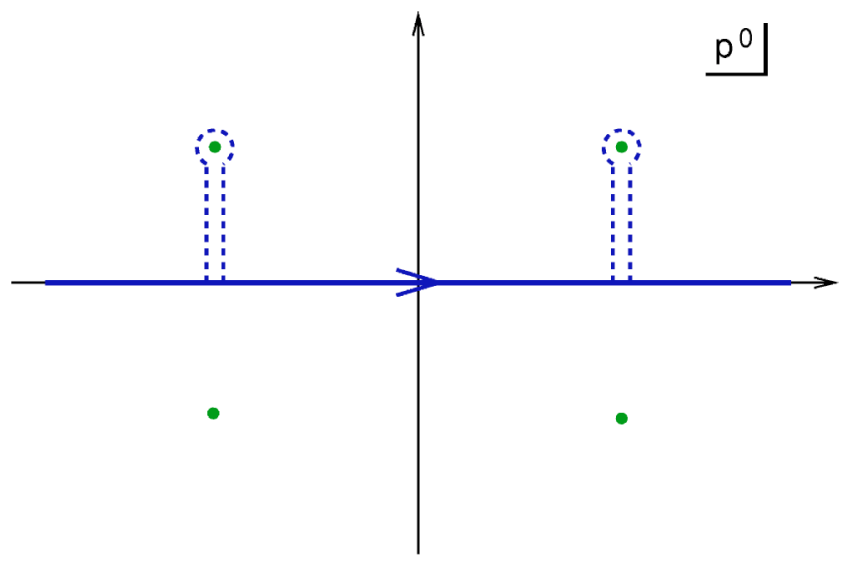

FIG. 2. Representation in the complex plane of the values of $p^{0}$ for which the coefficient of the semiclassical Einstein equation for the tensorial components of the Einstein tensor in Fourier space vanishes. The case $\mu<\mu_{\text {crit }}$ and a particular value of $\vec{p}^{2}$ were considered, but the qualitative structure will remain the same for any other value of $\vec{p}^{2}$. The plot also corresponds to the structure of the poles of the tensorial part of the propagator $\widetilde{D}_{\mu \nu \alpha \beta}(p)$. The solid line corresponds to the integration contour when Fourier transforming back to spacetime coordinates which follows from the prescription proposed by Hawking et al. [64] and was chosen in Ref. [54]. Changing this contour as indicated by the dashed lines, one obtains a strictly retarded propagator, but it exhibits exponential instabilities for large positive time differences associated with the two poles on the upper half of the complex plane.

[21]. The two zeros on the upper half of the complex plane correspond to solutions in spacetime coordinates exponentially growing in time, whereas the two on the lower half correspond to solutions exponentially decreasing in time. Strictly speaking, these solutions only exist in spacetime coordinates, since their Fourier transform is not well defined. They are commonly referred to as runaway solutions and for $\mu \sim l_{p}^{-1}$ they grow exponentially in time scales comparable to the Planck time.

\section{Quantum mechanical systems with higher order time derivatives}

Before proceeding to discuss the situation in stochastic gravity, it is interesting to make a few remarks about the quantization of higher derivative theories and the counterparts of the previous classical instabilities in the quantum context. Let us consider first a free theory with a structure analogous to that of linearized semiclassical gravity around Minkowski spacetime without including the nonlocal terms. It is characterized by the following Lagrangian, which corresponds to a harmonic oscillator with a higher derivative term:

$$
L(q, \dot{q}, \ddot{q})=\frac{\tau}{2} \ddot{q}^{2}+\frac{1}{2} \dot{q}^{2}-\frac{1}{2} \Omega^{2} q^{2}
$$

To begin with, one can consider a generalization of the usual canonical formalism introduced by Ostrogradski to deal with theories involving higher order derivatives (see, for instance
Refs. $[19,77])$. The theory can then be quantized following the standard canonical quantization rules. The corresponding Wigner function (or Wigner functional if a field theory were considered) can also be introduced. The pathological character of the theory becomes clear by diagonalizing the Hamiltonian and realizing that the result corresponds to two independent harmonic oscillators, but with one of them having a negative sign in the kinetic term. For $\tau<0$ the potential term of the harmonic oscillator with the negative kinetic term is also negative and the classical solutions do not exhibit instabilities. However, in any case the configurations for the harmonic oscillator with the negative kinetic term can have negative energies arbitrarily large in absolute value. Moreover, the frequency for that oscillator is proportional to $\tau^{-1 / 2}$ and diverges as $\tau \rightarrow 0$. At the quantum level, such a theory also gives rise to negative eigenvalues of the Hamiltonian arbitrarily large in absolute value, but can be alternatively formulated in terms of a Hamiltonian without negative energies by introducing states with negative norm (commonly referred to as ghosts) [65]. This fact is often argued in a qualitative way by pointing out that the propagator of the theory in Fourier space is proportional to

$$
\frac{1}{\omega^{2}-\Omega^{2}}-\frac{1}{\omega^{2}+\tau^{-1}} .
$$

It should also be mentioned that Hawking and Hertog have suggested a prescription for dealing with that kind of theories which is based on imposing well-defined boundary conditions in Euclidean time and then Wick rotating back to Lorentzian time. The results have then a nonsingular limit $\tau \rightarrow 0$, so that when the higher order derivative term in the Lagrangian is small, one essentially recovers the results of the second order theory [65].

Even though there is a range of parameters $(\tau<0)$ in which the free theory described above does not exhibit instabilities, they arise when a nonlinear self-interaction term is added to the Lagrangian. The reason is that the two Hamiltonian contributions corresponding to a couple of harmonic oscillators, one with a negative energy spectrum and the other with a positive one, can have a stable evolution as long as they are decoupled. However, adding an interaction term couples them in such a way that one can acquire negative energies arbitrarily large in absolute value while the other gains large positive energies, which is the source of instability. In general this is reflected in the structure of the propagator as a shift of the poles on the real axis to the complex plane. Hawking et al. have argued that well-behaved results can still be obtained by imposing boundary conditions which discard solutions which grow unboundedly in time $[64,65]$. Those conditions can be implemented by a suitable choice of the integration contour on the complex plane when computing the inverse Fourier transform of the propagator, but causality is violated at small time scales (we will come back to this point below). Another possibility, when the parameter $\tau$ is small, is to make use of an order reduction procedure $[21,22,78]$, which consists of differentiating the equation of 
motion with respect to time, substituting back into the original equation and discarding the terms of higher order in $\tau$. This procedure can be iterated as many times as necessary to get a second order equation valid up to the corresponding order of $\tau$. The usual canonical formalism associated with the second order equation of motion can then be employed to evolve the Wigner function. It should be stressed that, although we have considered a simple model as an illustrating example, the previous methods have been applied to more involved situations, including SCG $[22,21]$ and quantum cosmology [64].

\section{Runaway solutions in stochastic gravity}

Let us now address the case of stochastic gravity and see how the instabilities in SCG and the difficulties in quantizing theories with higher order derivatives are related. First of all, we recall that in Appendix $\mathrm{C}$ the counterterms quadratic in the curvature were ignored and it was implicitly assumed that the Einstein-Langevin equation was a second order integro-differential equation whose initial conditions were completely determined by specifying the metric perturbation and its normal derivative on the initial Cauchy hypersurface. If the counterterms quadratic in the curvature, which give rise to higher order derivative terms, are also taken into account, the generalized canonical formalism referred to above and the corresponding Wigner functional should be used. In fact, as a result of the singular behavior of the nonlocal part of the dissipation kernel at the initial time, specifying initial conditions at a finite initial time is an even more delicate matter. In any case, since we have to consider asymptotic initial conditions to deal with the singular coincidence limit of the noise kernel, as explained in Appendix D, we do not need to be concerned about the problems associated with finite initial times. Runaway solutions, however, still exist and some method to deal with them is required. In particular, when computing two-point correlation functions in the context of stochastic gravity, the existence of runaway solutions has implications for both the intrinsic and the induced contributions.

One possible method for dealing with the existence of runaway solutions is the order reduction prescription. As explained above, the method is based on treating perturbatively the terms involving higher order derivatives, differentiating the equation under consideration and substituting back the higher derivative terms in the original equation keeping only terms up to the required order in the perturbative parameter. In the case of the semiclassical Einstein equation, the perturbative parameter employed is $\hbar$ or, equivalently, the square of the Planck length $l_{p}^{2}=\kappa / 8 \pi$. If we consider the semiclassical Einstein equation for linear metric perturbations around Minkowski spacetime and differentiate twice with respect to the background covariant derivative, it becomes clear that the second order derivatives of the Einstein tensor are of order $\kappa$. Substituting back into the original equation, we get the following equation up to order $\kappa^{2}$ :

$$
G_{a b}^{(1)}[g+h]=0+O\left(\kappa^{2}\right),
$$

where no effects from the vacuum polarization of the quantum matter fields are left. Since the linearized semiclassical Einstein equation coincides with the homogeneous part of the Einstein-Langevin equation, Eq. (E7) governs the contribution of the intrinsic fluctuations to the quantum correlation function, which coincides with that of free gravitons. Similarly, when making use of the order reduction prescription, the Einstein-Langevin equation becomes

$$
G_{a b}^{(1)}[g+h]=\kappa \xi_{a b}+O\left(\kappa^{2}\right),
$$

where the stochastic source, whose correlation function only depends on the background metric and hence does not involve higher order derivatives of the metric perturbation, is not affected by the order reduction procedure. Therefore, in contrast to the intrinsic fluctuations, there will still be a nontrivial contribution to the induced fluctuations due to the polarization of the quantum matter fields, but no contribution from the dissipation kernel is left in the Einstein-Langevin equation. Since all the terms involving higher order derivatives, which were associated with the dissipation kernel, have been discarded, an ordinary Wigner functional can be introduced without any need to consider generalized Ostrogradski momenta. Furthermore, the absence of the dissipation kernel also allows the possibility of specifying initial conditions at a finite initial time as far as the homogeneous solutions (relevant for the computation of the intrinsic fluctuations) and the retarded propagator are concerned. Nevertheless, one is still forced to consider asymptotic initial conditions in order to get a finite result for the induced fluctuations due to the singular coincidence limit of the noise kernel, as explained in the previous appendix.

Hawking $e t a l$. have proposed an alternative procedure for dealing with the runaway solutions $[64,65]$. Their method is based on imposing final boundary conditions which discard those solutions that grow unboundedly in time. Let us first see how their approach can be applied to the computation of the intrinsic fluctuations by considering the particular case of a massless and conformally coupled scalar field. From Eq. (E4) and Fig. 2 one can see that, in addition to the solution $G_{\mu \nu}^{(1)}=0$, the solutions of the tensorial part grow or decrease exponentially in time. The exponentially growing solutions are discarded when the final boundary condition is imposed, and the contributions from the exponentially decreasing ones also vanish if regular initial conditions are specified at an asymptotic initial time. On the other hand, from Eq. (E3) one can see that the situation is analogous for the solutions of the scalar part when $\bar{\beta}<0$. For $\bar{\beta}>0$ the solutions are oscillatory and, hence, are not discarded when the final boundary condition is imposed (in contrast to the situation where the order reduction prescription is used).

Let us now apply the previous approach to the computation of the induced fluctuations. When considering asymptotic initial conditions, the relevant propagator for expressing the linearized Einstein tensor in terms of the sto- 
chastic source, can be obtained by inverting $F_{\mu \nu \alpha \beta}(p)$ in Eq. (7). The resulting propagator, $\widetilde{D}_{\mu \nu \alpha \beta}(p)$, exhibits a number of poles in the complex plane, as illustrated in Fig. 2. The expression for the retarded propagator in spacetime coordinates corresponds to choosing the integration path represented by the dashed line in Fig. 2 when Fourier transforming back from momentum space. It exhibits the appropriate causal behavior: $D_{\mu \nu \alpha \beta}(x-y)=0$ for $t_{x}<t_{y}$, as can be seen by closing the integration contour on the upper half of the complex plane. However, for $t_{x}>t_{y}$ it increases exponentially in time due to the contributions from the two poles on the upper half of the complex plane when closing the path on the lower half. Imposing the final boundary conditions which discard solutions growing unboundedly in time is equivalent to taking a different integration path: that represented by a solid line in Fig. 2. The resulting propagator does not exhibit exponential instabilities, but gives rise to causality violations since $D_{\mu \nu \alpha \beta}(x-y) \neq 0$ for $t_{x}<t_{y}$ (the characteristic timescale of these causality violations is of order $\sqrt{N} l_{p}$ ). This propagator is the only one which has a well-defined Fourier transform. It was employed in Ref. [54], where it was argued that any other propagator should yield an equivalent result for the correlation function obtained by solving the EinsteinLangevin equation. This argument is certainly true for propagators with a well-defined Fourier transform. However, the existence of poles off the real axis gives rise to propagators in spacetime coordinates (they do not have a well-defined Fourier transform because of the exponentially growing or decreasing contributions) which yield inequivalent results for the correlation function. Since this choice for the propagator was made, the results obtained in Ref. [54] correspond to those that would follow when employing the procedure proposed by Hawking et al. In fact, Hawking et al. applied their method to quantum propagators, but, as we have described, it can also be used when solving the semiclassical Einstein equation and the Einstein-Langevin equation. The stochastic correlation functions obtained are then equivalent to the quantum correlation functions (CTP propagators) which would result from the application of the prescription.

\section{Estimates of radiative corrections for a single matter field and a large number of them}

SCG is expected to provide reliable results as long as the characteristic length scales under consideration are much larger than the Planck length $l_{p}$ [21]. This can be qualitatively argued by estimating the magnitude of the different contributions to the effective action (considering the relevant Feynman diagrams and using dimensional arguments): the Einstein-Hilbert term and the radiative quantum corrections. The Einstein-Hilbert term is of order $l_{p}^{-2} R$ (the characteristic curvature $R$ is simply given by $L^{-2}$, where $L$ is the characteristic length scale of our problem), the vacuum polarization terms involving loops of matter fields are of order $R^{2}$, and higher loop corrections involving internal graviton propaga- tors are of order $l_{p}^{2} R^{3}$ or higher. Thus, we see that the higher order corrections not included in SCG are negligible provided that $L \gg l_{p}$. In that regime, however, the vacuum polarization terms only yield a small correction to the EinsteinHilbert term and any classical gravitational source which were present. The justification of the order reduction prescription is actually based on this fact. Therefore, significant effects from the vacuum polarization of the matter fields are only expected when their small corrections accumulate in time, as would be the case, for instance, for an evaporating macroscopic black hole all the way before reaching Planckian scales.

The previous estimates for the different terms in the effective action change in a remarkable way when a large number of fields, $N$, is considered. ${ }^{16}$ The vacuum polarization terms involving loops of matter become of order $N R^{2}$ and, similarly, the higher loop corrections involving internal graviton propagators are of order $N l_{p}^{2} R^{3}$ or higher (the contributions corresponding to one and two graviton loops are, respectively, of order $R^{2}$ and $l_{p}^{2} R^{3}$, but are negligible as compared to those from matter loops when $N$ is large). There is then a regime in which the vacuum polarization of the matter fields and the Einstein-Hilbert term are comparable when $L$ $\sim \sqrt{N} l_{p}$. On the other hand, the higher loop corrections will still be much smaller if $L \gg l_{p}$. Both conditions are compatible provided that the number of fields, $N$, is very large. This is, in fact, the kind of situation considered in trace anomaly driven inflationary models [64], such as that originally proposed by Starobinsky [63], where the exponential inflation is driven by a large number of massless conformal fields. The order reduction prescription would completely discard the effect from the vacuum polarization of the matter fields even though it is comparable to the Einstein-Hilbert term. In contrast, the procedure proposed by Hawking et al. keeps the contribution from the matter fields.

We conclude this appendix by mentioning that it has been pointed out that a similar kind of instability, which is closely connected to the existence of the Landau pole, is also present in scalar QED (as well as ordinary QED) [17,18,34]. Nevertheless, a number of nonperturbative studies on the evolution of the expectation value of the electromagnetic field using a large $N$ expansion have been carried out. In fact, it was suggested in Ref. [45] that by introducing a finite 3-momentum cutoff and considering a running coupling constant small enough at low energies, the problem with the Landau pole could be circumvented (at least from a practical point of view). Yet it seems unlikely that a similar procedure could work for the gravitational case due to the existence of higher derivatives. Moreover, introducing a 3-momentum cutoff would break general covariance and that would pose serious difficulties when implementing a consistent and natural renormalization scheme in general curved spacetimes.

\footnotetext{
${ }^{16}$ The actual physical Planck length $l_{p}$ is considered, not the rescaled one, $\sqrt{\kappa / 8 \pi}$, which is related to $l_{p}$ by $8 \pi l_{p}^{2}=\kappa=\bar{\kappa} / N$.
} 
[1] B.L. Hu and E. Verdaguer, Class. Quantum Grav. 20, R1 (2003).

[2] B.L. Hu and E. Verdaguer, Living Rev. Rel. 7, 3 (2004).

[3] P.R. Anderson, C. Molina-París, and E. Mottola, Phys. Rev. D 67, 024026 (2003).

[4] L.H. Ford, Ann. Phys. (N.Y.) 144, 238 (1982).

[5] Chung-I Kuo and L.H. Ford, Phys. Rev. D 47, 4510 (1993).

[6] L.H. Ford, Int. J. Theor. Phys. 38, 2941 (1999).

[7] L.H. Ford and C.H. Wu, Int. J. Theor. Phys. 42, 15 (2003).

[8] J. Borgman and L.H. Ford, gr-qc/0307043.

[9] L.H. Ford and N.F. Svaiter, Phys. Rev. D 56, 2226 (1997).

[10] H. Yu and L.H. Ford, Phys. Rev. D 60, 084023 (1999).

[11] H. Yu and L.H. Ford, Phys. Lett. B 496, 107 (2000).

[12] B.L. Hu, gr-qc/9511077.

[13] B.L. Hu, gr-qc/9607070.

[14] B.L. Hu, Int. J. Theor. Phys. 38, 2987 (1999).

[15] B.L. Hu, Int. J. Theor. Phys. 41, 2091 (2002).

[16] G.T. Horowitz, Phys. Rev. D 21, 1445 (1980).

[17] G.T. Horowitz, in Quantum Gravity 2: A second Oxford symposium, edited by C.J. Isham, R. Penrose, and D.W. Sciama (Clarendon Press, Oxford, 1981).

[18] R.D. Jordan, Phys. Rev. D 36, 3593 (1987).

[19] J.Z. Simon, Phys. Rev. D 41, 3720 (1990).

[20] J.Z. Simon, Phys. Rev. D 43, 3308 (1991).

[21] E.E. Flanagan and R.M Wald, Phys. Rev. D 54, 6233 (1996).

[22] L. Parker and J.Z. Simon, Phys. Rev. D 47, 1339 (1993).

[23] N.C. Tsamis and R.P. Woodard, Nucl. Phys. B474, 235 (1996).

[24] N.C. Tsamis and R.P. Woodard, Phys. Rev. D 54, 2621 (1996).

[25] N.C. Tsamis and R.P. Woodard, Ann. Phys. (N.Y.) 253, 1 (1997).

[26] N.C. Tsamis and R.P. Woodard, Phys. Rev. D 57, 4826 (1998).

[27] B.L. Hu and N.G. Phillips, Int. J. Theor. Phys. 39, 1817 (2000).

[28] N.G. Phillips and B.L. Hu Phys. Rev. D 62, 084017 (2000).

[29] E. Calzetta and B.L. Hu, Phys. Rev. D 61, 025012 (2000).

[30] C.W. Misner, K.S. Thorne, and J.A. Wheeler, Gravitation (Freeman, San Francisco, 1973).

[31] R.M. Wald, General Relativity (The University of Chicago Press, Chicago, 1984).

[32] N.D. Birrell and P.C.W. Davies, Quantum Fields in Curved Space (Cambridge University Press, Cambridge, England, 1994).

[33] R.M. Wald, Quantum Field Theory in Curved Spacetime and Black Hole Thermodynamics (The University of Chicago Press, Chicago, 1994).

[34] J.B. Hartle and G.T. Horowitz, Phys. Rev. D 24, 257 (1981).

[35] J. Schwinger, J. Math. Phys. 2, 407 (1961).

[36] P.M. Bakshi and K.T. Mahanthappa, J. Math. Phys. 4, 1 (1963).

[37] L.V. Keldysh, Zh. Éksp. Teor. Fiz 47, 1515 (1964) [Sov. Phys. JETP 20, 1018 (1965)].

[38] K. Chou, Z. Su, B. Hao, and L. Yu, Phys. Rep. 118, 1 (1985).

[39] R.D. Jordan, Phys. Rev. D 33, 444 (1986).

[40] E. Calzetta and B.L. Hu, Phys. Rev. D 35, 495 (1987).
[41] A. Campos and E. Verdaguer, Phys. Rev. D 49, 1861 (1994).

[42] E. Calzetta and B.L. Hu, Phys. Rev. D 49, 6636 (1994).

[43] B.L. Hu and A. Matacz, Phys. Rev. D 51, 1577 (1995).

[44] E. Calzetta, A. Campos, and E. Verdaguer, Phys. Rev. D 56, 2163 (1997).

[45] F. Cooper, S. Habib, Y. Kluger, E. Mottola, J.P. Paz, and P.R. Anderson, Phys. Rev. D 50, 2848 (1994).

[46] R. Kubo, Phys. Rev. D 29, 255 (1966).

[47] E. Mottola, Phys. Rev. D 33, 2136 (1986).

[48] A. Campos and B.L. Hu, , Phys. Rev. D 58, 125021 (1998).

[49] R.P. Feynman and F.L. Vernon, Ann. Phys. (N.Y.) 24, 118 (1963).

[50] R.P. Feynman and A.R. Hibbs, Quantum Mechanics and Path Integrals (McGraw-Hill, New York, 1965).

[51] R. Martín and E. Verdaguer, Phys. Rev. D 60, 084008 (1999).

[52] R. Martín and E. Verdaguer, Int. J. Theor. Phys. 38, 3049 (1999).

[53] W. Tichy and E.E. Flanagan Phys. Rev. D 58, 124007 (1998).

[54] R. Martín and E. Verdaguer, Phys. Rev. D 61, 124024 (2000).

[55] B.L. Hu and S. Sinha, Phys. Rev. D 51, 1587 (1995).

[56] A. Campos and E. Verdaguer, Phys. Rev. D 53, 1927 (1996).

[57] R. Martín and E. Verdaguer, Phys. Lett. B 465, 113 (1999).

[58] A. Roura and E. Verdaguer (in preparation).

[59] E. Calzetta, A. Roura, and E. Verdaguer, Physica A 319, 188 (2003).

[60] J.D. Jackson, Classical Electrodynamics (Wiley, New York, 1999).

[61] P.R. Johnson and B.L. Hu, Phys. Rev. D 65, 065015 (2002).

[62] J.Z. Simon, Phys. Rev. D 45, 1953 (1992).

[63] A.A. Starobinsky, Phys. Lett. 91B, 99 (1980).

[64] S.W. Hawking, T. Hertog, and H.S. Reall, Phys. Rev. D 63, 083504 (2001).

[65] S.W. Hawking and T. Hertog, Phys. Rev. D 65, 103515 (2002).

[66] M.T. Jaekel and S. Reynaud, Ann. Phys. (Leipzig) 4, 68 (1995).

[67] E. Tomboulis, Phys. Lett. 70B, 361 (1977).

[68] A. Roura and E. Verdaguer (in preparation).

[69] C.H. Wu and L.H. Ford, Phys. Rev. D 64, 045010 (2001).

[70] C.H. Wu, Chung-I Kuo, and L.H. Ford, Phys. Rev. A 65, 062102 (2002).

[71] M. Hillary, R.F. O'Connell, M.O. Scully, and E.P. Wigner, Phys. Rep. 106, 121 (1984).

[72] A. Roura, Ph.D. thesis, Universitat de Barcelona, 2001.

[73] L. Schwartz, Théory des Distributions (Hermann, Paris, 1957).

[74] A.H. Zemanian, Distribution Theory and Transform Analysis (Dover, New York, 1987).

[75] H. Grabert, P. Schramm, and G. Ingold, Phys. Rep. 168, 115 (1988).

[76] B.L. Hu, J.P. Paz, and Y. Zhang, Phys. Rev. D 45, 2843 (1992).

[77] F.J. de Urries and J. Julve, J. Phys. A 31, 6949 (1998).

[78] L.D. Landau and E.M. Lifshitz, The Classical Theory of Fields, Course of Theoretical Physics, Vol. 2 (Pergamon Press, Oxford, 1975).

[79] J.M. Pons, Gen. Relativ. Gravit. 35, 147 (2003). 